Yihan Zhang

Ping Wang

Yachong Liu

Jingfeng $\mathrm{Hu}$

http://dx.doi.org/10.21278/brod72307

ISSN 0007-215X

eISSN 1845-5859

\title{
NONLINEAR ROLLING STABILITY AND CHAOS RESEARCH OF TRIMARAN VESSEL WITH VARIABLE LAY-OUTS IN REGULAR AND IRREGULAR WAVES UNDER WIND LOAD
}

\author{
UDC 629.5.015.13:629.5.015.141:629.5.017.22:629.5.022.3
}

Original scientific paper

\begin{abstract}
Summary
The trimaran vessel rolls strongly at low forward speed and may capsize in high sea conditions due to chaos and loss of stability, which is not usually considered in conventional limit-based criteria. In order to perfect the method of measuring roll performance of trimaran, a set of nonlinear roll motion stability analysis method based on Lyapunov and Melnikov theory was established. The nonlinear roll motion equation was constructed by CFD and high-order polynomial fitting method. The wave force threshold of rolling chaos in regular waves is calculated by Gauss-Legendre numerical integration method. The limited significant wave height of rolling chaos in random sea conditions is deduced by the phase space transfer rate, and the complex effect of wind load is superposed in the calculation. The influence of trimaran configuration on the roll system is analyzed through the state differentiation of homoclinic and heteroclinic orbit in phase portrait. The calculation of the maximum Lyapunov exponent further verified the applicability of Melnikov method, and the topological structure change of gradual failure of the rolling system is analyzed by the erosion of safe basin. The complex changes of the nonlinear damping coefficient and the nonlinear restoring moment coefficient caused by the change of the transverse lay-outs between the main hull and side hull have a significant influence on chaos and stability, and the existence of wind load has a certain weakening effect on the stability and symmetry of the system. The conclusion also further indicates the importance of the lay-outs to the dynamic stability of the trimaran vessel, which is significant for its seakeeping design.
\end{abstract}

Key words: $\quad$ Trimaran; Nonlinear rolling stability; Transverse Lay-outs; Melnikov function; Lyapunov exponent; Phase space transfer rate

\section{Introduction}

In Naval Architecture, "Stability" has a wide meaning, which usually involves static and dynamic stability, and its fundamentals have wider implications for the design and operation of ships [1]. In recent years, with the continuous development of second-generation stability, the research on dynamic stability has been increasing for mono-hull ships. However, the method 
Yihan Zhang, Ping Wang, Yachong Liu, Jingfeng Hu.
Nonlinear rolling stability and chaos research of trimaran vessel with variable lay-outs in regular and irregular waves under wind load

of limiting bounds is still used for the calibration of roll motion in the design of the trimaran vessel, and it is necessary to study its dynamic rolling stability.

Rolling system can be considered as a softening duffing system, and the stability is a nonlinear dynamic problem [2-3]. In the field of dynamic rolling stability, there are two main approaches, for regular wave and irregular wave, it can be divided into deterministic method and stochastic method. The rolling stability of the deterministic system refers to the roll motion in regular waves, and the coefficients in the system are considered to be fixed, and the methods mainly include catastrophe theory, Floquet theory, Melnikov function, fractal dimension, Kolmogorov entropy and safe basin [4-6]. Virgin [7-9] studied an oscillator by the method of harmonic balance and then the nonlinear roll motion stability of vessel in regular waves and its chaotic phenomena were studied, and he pointed out that ships usually went through a chaotic stage before capsizing, and he gave a method to predict this chaotic motion.

In 1963, Melnikov proposed a method to measure the distance between stable and unstable manifolds after splitting while studying the perturbation of homoclinic and heteroclinic orbits of conservative systems. Holmes and Greenspan discussed the averaging and chaotic motions and homoclinic bifurcation of some oscillator by Melnikov method [10-11], and Thompson [12] applied Melnikov method to study ship capsizing for the first time in 1987. Later on, it gradually became the most mainstream method. Falzarano et al. [13] introduced the Melnikov method into the study of ship motion and analyzed the homoclinic bifurcation and heteroclinic bifurcation. Nayfeh and Balachandran [14] studied the dynamic stability of various quantitative nonlinear systems systematically by Melnikov method and Lyapunov exponent. In recent years, Maki studied a non-Hamiltonian exact heteroclinic orbit by Melnikov integral formula [15]. Wan [16] used Melnikov method to study the stability of roll motion under the condition of large damping. Surendran et al. [17] used Melnikov method to study roll motion stability of a RORO ship.

The sea that ships actually sail is composed of random waves, which are usually called irregular waves in the study of seakeeping. For linear motion system, random waves are usually assumed to be stationary random processes of all states of ergodic when predicting the motion performance in irregular waves based on spectral analysis method. However, this method is not applicable to the strong-nonlinear roll motion. With the development of stochastic theory, some scholars applied the method of probability and statistics theory and transform the problem into the solution of FPK(Fokker-Planck-Kolmogorov) equation to satisfy Markov process [18]. The probability density is obtained by solving FPK equation, and then the stability of roll motion can be judged. Nekrasov and Haddara first used the Markov process to study ship roll motion, but only limited to white noise excitation and small or medium amplitude roll motion [19-20]. Roberts [21] used the mean equation to reduce the FPK equation from two dimensions to one dimension, and at the same time considered the nonlinear roll damping and nonlinear restoring moment to overcome the limitation of white noise excitation. Subsequently, he carried out a lot of improved work [22-23], and he perfected the theoretical basis for solving the stability of roll motion under irregular sea conditions based on FPK equation, which was widely used by scholars in the future.

Chai used the path integral method to study the effect of the white noise processed by the high-order filter on the roll stability [24-25]. Maki used non-gaussian probability density function to study the capsizing probability in irregular beam seas [26]. Ren used the path integral method to study the first passage of the nonlinear rolling motion of a mono-hull ship in random sea conditions under ice load [27]. However, the limitations of Markov process, such as gaussian white noise assumption and nonlinear intensity, lead to some deficiencies in the prediction accuracy and practical application. The random Melnikov theory is gradually developed by referring to Melnikov function in deterministic methods. Based on the random 
Melnikov process, Li studied the rolling motion stability of a mono-hull ship in random sea conditions and analyzed the influence of parameters on the safe basin [28]. Frey and Jiang first proposed to judge the stability of random rolling based on the random Melnikov theory through the phase space transfer rate, and he defined the phase space transfer rate as the area from the safe domain to the unsafe domain within a unit period [29-30]. Subsequently, it has been widely used in the study of random roll stability of ships, but mainly concentrated on mono-hull ships. Liu studied the phase - space transfer rate of random wave ship nonlinear rolling under green water [31]. Zhang studied the change of phase space transfer rate with wave direction and forward speed [32].

At present, there are few studies on the roll stability of multi-hulled ships, such as trimaran vessel, in either regular wave or random sea conditions. The trimaran vessel, as a popular multihull ship type in the last two decades, its configuration is more flexible than single-hull ship, and the designer can get a better scheme by changing the transverse and longitudinal lay-outs between the main hull and the side hull [33-35]. As we all know, with the increasing of transverse spacing, the trimaran vessel has a larger radius of transverse meta-centering and better stability than mono-hull ship in static stability, but the roll period is reduced quickly. The rolling system is a nonlinear system by the function of the nonlinear of rolling damping and restoring moment. In addition to the conventional component such as friction damping, wavemaking damping, vortex damping, and appendage damping, nonlinear damping caused by flow interference between the main hull and the side hull should be considered for the trimaran vessel [36]. The nonlinearity of the restoring moment coefficient is mainly related to the irregularity of the hull, which is also more prominent for the trimaran vessel. Under severe sea conditions and low forward speed, this kind of ship will have a large nonlinear roll motion [37-38]. The change of the coefficient caused by the change of the lay-outs will cause some uncertainties to the stability. So, it is necessary to establish the method to study the rolling stability for trimaran vessels. This paper focuses on the establishment of roll motion stability prediction methods for trimaran vessel in complex environments with regular and irregular waves and wind loads under the variation of transverse layouts.

\subsection{Principal Dimensions}

A 600-ton high-speed transportation trimaran vessel is adopted as the research object. Table 1 shows the principal dimensions. The characteristics of different transverse spacing from $4.5 \mathrm{~m}$ to $10 \mathrm{~m}$ will be studied. When the spacing changes, the draft, displacement, and center of gravity remain unchanged, but the hydrodynamic coefficients are changing, and the detailed data is shown in table 1 and subsequent studies. Fig. 1 shows the whole model and section lines when transverse spacing is $7.0 \mathrm{~m}$.

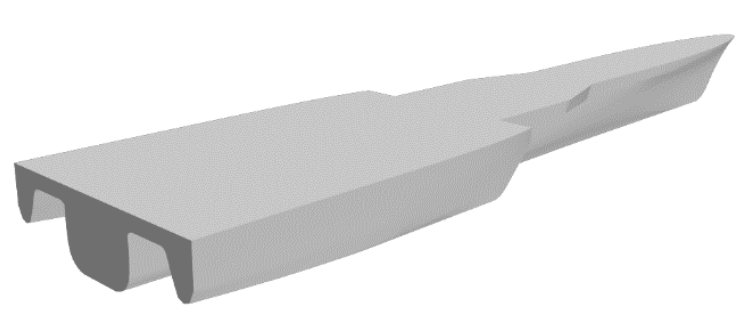

(a) 3D calculation model

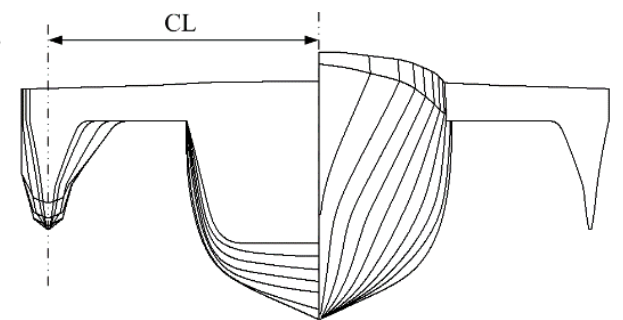

(b) Ship lines of cross section

Fig.1 Trimaran model 
Yihan Zhang, Ping Wang, Yachong Liu, Jingfeng $\mathrm{Hu}$.
Nonlinear rolling stability and chaos research of trimaran vessel with variable lay-outs in regular and irregular waves under wind load

Table1 Principal dimensions

\begin{tabular}{|c|c|c|c|}
\hline Item & Paticulars & Unit & Trimaran \\
\hline Length between perpendicular (main hull) & Lppm & $\mathrm{m}$ & 65 \\
\hline Moulded Breadth (main hull) & $B m$ & $\mathrm{~m}$ & 5.5 \\
\hline Draft (main hull) & $T m$ & $\mathrm{~m}$ & 2.8 \\
\hline Displacement (main hull) & $\Delta m$ & ton & 580 \\
\hline Length between perpendicular (side hull) & $L p p s$ & $\mathrm{~m}$ & 26 \\
\hline Moulded Breadth (side hull) & $B s$ & $\mathrm{~m}$ & 1.5 \\
\hline Draft (side hull) & $T s$ & $\mathrm{~m}$ & 1 \\
\hline Displacement (side hull) & $\Delta s$ & ton & 10 \\
\hline Longitudinal Spacing & $S L$ & $\mathrm{~m}$ & 0 (tail alignment) \\
\hline Transverse Spacing & $C L$ & $\mathrm{~m}$ & $4.5-10$ \\
\hline
\end{tabular}

1.2 Roll motion equation

$$
\left(J_{\varphi \varphi}+\Delta J_{\varphi \varphi}\right) \ddot{\varphi}+M(\dot{\varphi})+M(\varphi)=F \text { wave }(t)+F \text { wind }(t)
$$

The ship roll motion equation in waves can be expressed as equation (1). Where, $J_{\varphi \varphi}, \Delta J_{\varphi \varphi}$ are the transverse moment of inertia and the additional moment of inertia, respectively. $M(\dot{\varphi})$ is damping moment, $M(\varphi)$ is restoring moment, $F$ wave $(t)$ is wave force, $F$ wind $(t)$ is wind force.

\subsubsection{Nonlinear roll damping model}

Roll damping is always obtained by roll decay motion in still water which can be simulated by CFD or model experiments [39-40]. Due to the limited test conditions, one roll decay model test of the transverse spacing $\mathrm{CL}=7 \mathrm{~m}$ is carried out as shown in Fig.2, and other conditions are simulated by CFD method. The implicit inconstant method is applied to solve the Reynolds Average Equations (RANS), while $k-\omega$ model is applied as the turbulence model. The overlapping mesh technique is used as shown in Fig.3. The CFD results are compared with experiment. It can be seen from Fig. 4 that the accuracy is well verified. Fig.6 shows the roll decay curves of different CLs. Preliminary observation shows that trimarans with large transverse spacing decay more quickly.

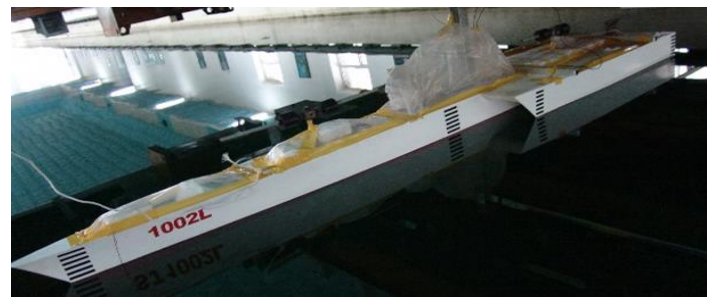

Fig. 2 Rolling decay model test

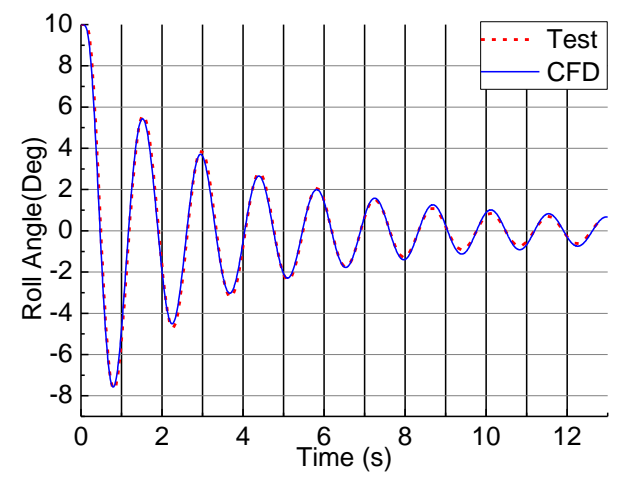

Fig.4 The comparison between CFD and model test

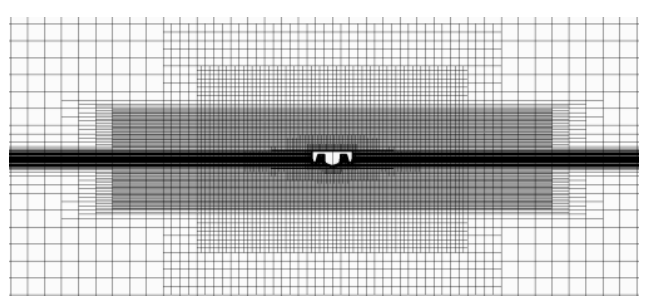

Fig. 3 CFD calculation mesh

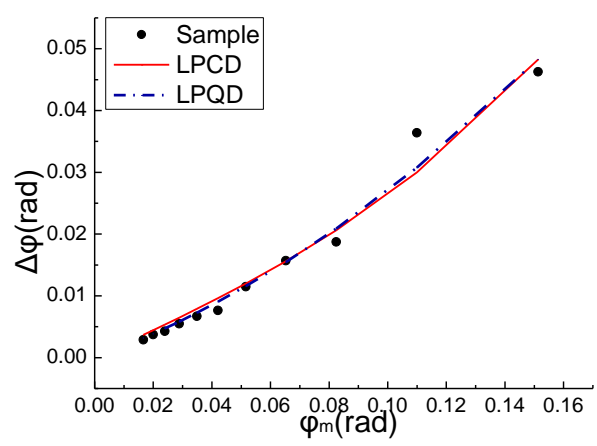

Fig.5 Comparison of $\varphi_{m}-\Delta \varphi$ curves of LPCD and LPQD damping models 


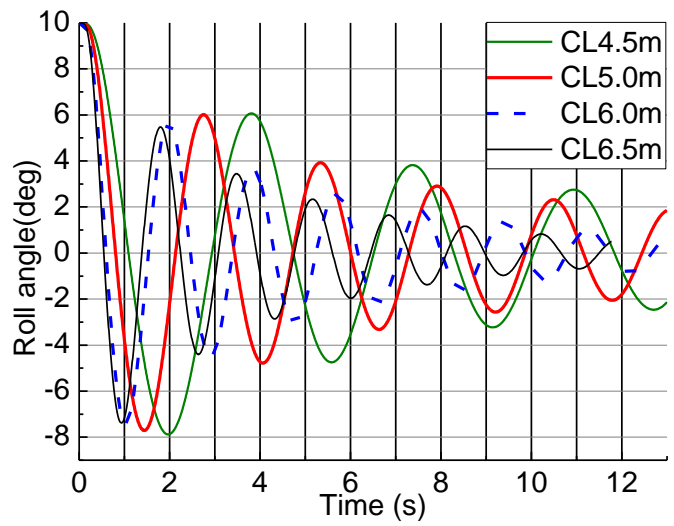

(a) $\mathrm{CL}=4.5 \mathrm{~m}-6.5 \mathrm{~m}$

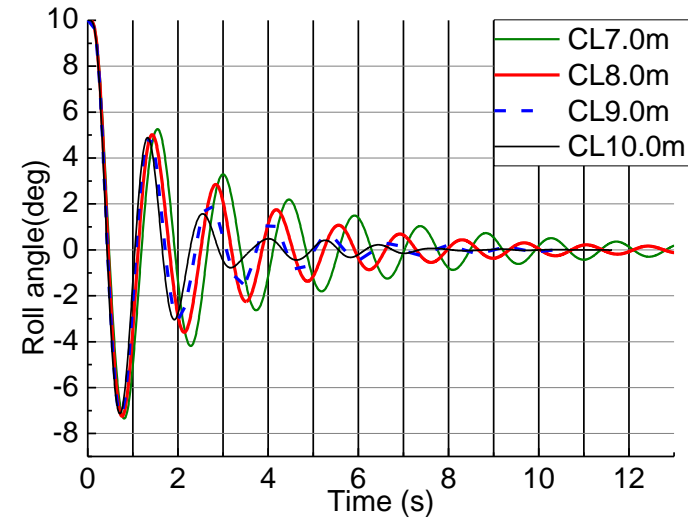

(b) $\mathrm{CL}=7 \mathrm{~m}-10 \mathrm{~m}$

Fig.6 Roll decay curves of different CLs

Similar to conventional mono-hull ships, the nonlinear roll damping moment $M(\dot{\varphi})$ of trimaran vessel can usually be expressed in linear plus square damping (LPQD) or linear plus cubic damping (LPCD), respectively shown as equation (2) and equation (3).

$$
\begin{aligned}
& M(\dot{\varphi})=A \dot{\varphi}+B \dot{\varphi}|\dot{\varphi}| \\
& M(\dot{\varphi})=A \dot{\varphi}+C \dot{\varphi}^{3}
\end{aligned}
$$

The selection of roll damping model may be different for different ship types. Wassermann [41] studied a post panama ship with different damping models and found that the linear plus square plus cubic model was more suitable. Bikdash et al. [42] studied the effect of damping models of a mono-hull ship, they derived an equivalent damping condition under which the two models yields the same Melnikov predictions. For trimaran vessels, Zhang [43] studied the roll damping in detail, and LPQD damping model was discussed at low speed while the LPCD damping model was not studied in his research. As we all know, the roll damping coefficient is usually obtained by using the $\varphi_{m}-\Delta \varphi$ curve according to the peak points of the decay curve, where, $\varphi_{m}$ and $\Delta \varphi$ are the mean values of the adjacent amplitudes and the difference of the adjacent amplitudes of the decay curve. In this paper, the two damping models were used to fit the $\varphi_{m}-\Delta \varphi$ curve in Fig.5, respectively, and it was found that the error between LPQD and LPCD models is very small within $0.5 \%$. Therefore, any of the two models can be used for the trimaran vessel. However, the LPQD model has the absolute item $|\dot{\varphi}|$, in order to deal with the equations conveniently for stability and chaotic analysis, LPCD model is adopted in following studies.

\subsubsection{Restoring moment model}

The direction of restoring moment is always opposite to the roll motion. In the case of small motion, the restoring moment has a linear relationship with the roll angle, but in the case of large roll motion, this relationship turns to nonlinear. In this paper, linear plus cubic plus quintic model is adopted to describe the nonlinear restoring moment, as shown in equation (4).

$$
\begin{aligned}
& M(\varphi)=C_{1} \varphi+C_{3} \varphi^{3}+C_{5} \varphi^{5} \\
& \text { It could also be written as } \quad M(\varphi)=M g\left(R_{1} \varphi+R_{3} \varphi^{3}+R_{5} \varphi^{5}\right)
\end{aligned}
$$

Where, $R_{1}, R_{3}, R_{5}$ are the lengths of restoring moment arm respectively, and can be obtained by fitting the GZ curve of a real ship. The GZ curves are obtained based on ship statics and corrected by free liquid level. $M g$ is the gravitational force or buoyancy. The fitting curve of restoring moment arm when the transverse spacing is $5 \mathrm{~m}$ and the transverse spacing is $8 \mathrm{~m}$ are shown in Fig.7 and Fig.8, indicating that the above nonlinear restoring moment model has a high precision. 
Yihan Zhang, Ping Wang, Yachong Liu, Jingfeng $\mathrm{Hu}$.
Nonlinear rolling stability and chaos research of trimaran vessel with variable lay-outs in regular and irregular waves under wind load

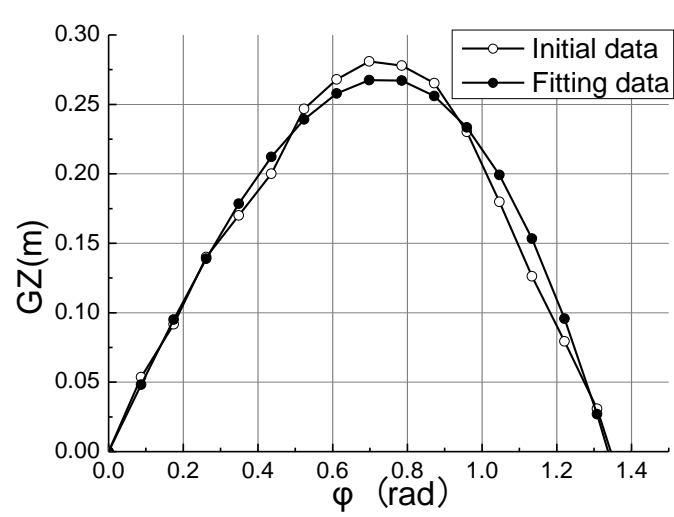

Fig.7 Nonlinear restoring moment arm fitting curve $(\mathrm{CL}=5.0 \mathrm{~m})$

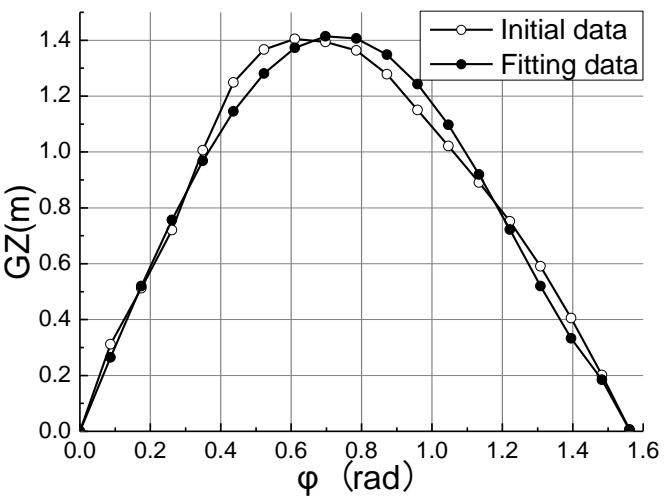

Fig.8 Nonlinear restoring moment arm fitting curve $(\mathrm{CL}=8.0 \mathrm{~m})$

\subsubsection{Model of wind load}

The model of wind load changing with roll angle can be defined as:

$$
F_{\text {wind }}=F_{0} \cos \varphi
$$

Where, $F_{0}$ is the wind force when roll angle is zero. For the sake of research, we can expand out $\cos \varphi$ by Taylor Expansion:

$$
F_{\text {wind }}=F_{0}\left(1-\frac{\varphi^{2}}{2}+\frac{\varphi^{4}}{24}\right)
$$

$F_{0}$ is mainly affected by the wind speed and shape above the waterline of the trimaran as shown in equation (8). The CFD method is used for calculation of the wind force coefficient $C_{y}$. Fig.9 is a scalar diagram of the wind field. The calculated wind load coefficient $C_{y}$ is 0.805 for this trimaran vessel. Then, according to different wind speeds, $F_{0}$ can be obtained.

$$
F_{0}=\frac{1}{2} C_{y} \rho V^{2} A \cdot l_{A}
$$

Where, $V$ is the velocity of wind, $A$ is windward area, $\rho$ is the air density, $l$ is the arm of the wind force.

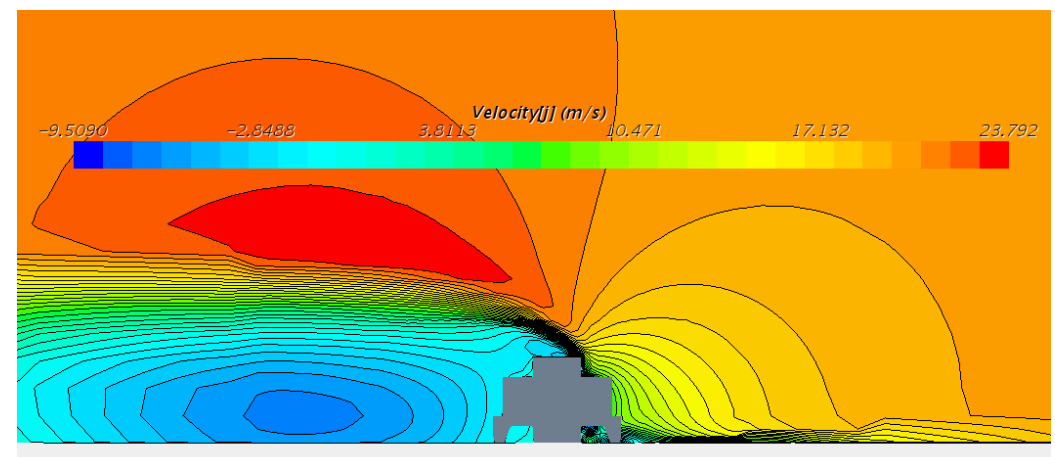

Fig. 9 Scalar diagram of the wind field

\subsubsection{Parameters of rolling model}

According to the above analysis, the nonlinear roll motion equation of the trimaran vessel in regular beam waves is obtained as follows:

$$
\left(J_{\varphi \varphi}+\Delta J_{\varphi \varphi}\right) \ddot{\varphi}+2 N_{1} \dot{\varphi}+N_{3} \dot{\varphi}^{3}+C_{1} \varphi+C_{3} \varphi^{3}+C_{5} \varphi^{5}=F(t)+F_{\text {wind }}
$$

Divide both sides by $J_{\varphi \varphi}+\Delta J_{\varphi \varphi}$, equation (9) is simplified to:

$$
\ddot{\varphi}+2 v_{\varphi \varphi} \dot{\varphi}+v_{3} \dot{\varphi}^{3}+r_{1} \varphi+r_{3} \varphi^{3}+r_{5} \varphi^{5}=f(t)+f \text { wind }
$$


Table2 Parameters at different transverse layouts of nonlinear roll system

\begin{tabular}{|c|c|c|c|c|c|}
\hline $\mathrm{CASE}$ & $2 v_{\varphi \varphi}$ & $v_{3}$ & $r_{1}$ & $r_{3}$ & $r_{5}$ \\
\hline & $\mathrm{s}^{-1}$ & $\mathrm{~s}$ & $\mathrm{~m}$ & $\mathrm{~s}^{-2}$ & $\mathrm{~s}^{-2}$ \\
\hline $\mathrm{Cl}=4.5 \mathrm{~m}$ & 0.055 & 5.675 & 0.188 & -0.134 & 0.003 \\
\hline $\mathrm{Cl}=4.8 \mathrm{~m}$ & 0.065 & 5.762 & 0.281 & -0.183 & 0.010 \\
\hline $\mathrm{Cl}=5.0 \mathrm{~m}$ & 0.065 & 6.438 & 0.356 & -0.238 & 0.022 \\
\hline $\mathrm{Cl}=5.5 \mathrm{~m}$ & 0.080 & 6.573 & 0.531 & -0.369 & 0.051 \\
\hline $\mathrm{Cl}=6.0 \mathrm{~m}$ & 0.101 & 5.314 & 0.689 & -0.488 & 0.079 \\
\hline $\mathrm{Cl}=6.5 \mathrm{~m}$ & 0.129 & 4.025 & 0.836 & -0.609 & 0.105 \\
\hline $\mathrm{Cl}=7.0 \mathrm{~m}$ & 0.155 & 3.292 & 0.976 & -0.723 & 0.131 \\
\hline $\mathrm{Cl}=8.0 \mathrm{~m}$ & 0.188 & 1.649 & 1.199 & -0.912 & 0.177 \\
\hline $\mathrm{Cl}=9.0 \mathrm{~m}$ & 0.301 & - & 1.401 & -1.084 & 0.216 \\
\hline $\mathrm{Cl}=10 \mathrm{~m}$ & 0.335 & - & 1.577 & -1.232 & 0.249 \\
\hline
\end{tabular}

Table 2 shows the detailed parameters in equation (10) at different transverse spacing. To study the transverse spacing as a variable, the center of mass and displacement are kept constant. As we can see, different CL has different nonlinear restoring moment coefficients $r_{1}, r_{3}, r_{5}$, which are fitted by corresponding GZ curves. With the increase of CL, the damping generally presents an increasing trend, with the linear damping increasing all the time, while the nonlinear damping increases first and then decreases. When the CL increases to $8 \mathrm{~m}$, the damping coefficient can only be fitted as linear damping, which may be due to the reduction of nonlinear interference caused by too large transverse spacing between main hull and side hull.

\section{Chaotic analysis and validation in regular waves}

\subsection{Phase portraits analysis}

The equilibrium point calculation and phase portraits analysis in the nonlinear dynamic system are usually taken as the key stages in whole stability analysis process. The stability of the autonomous system near the equilibrium point can be qualitatively analyzed by phase portrait of homoclinic or heteroclinic orbit, which is the basement and precondition for stability judgement of the non-autonomous system. Nayfeh and Balachandran [14] has provided the homoclinic and heteroclinic orbits and phase portraits research of some typical quantic nonlinear systems, which is useful for nonlinear roll system of the trimaran vessel.

We study the equation of roll motion under the action of regular beam waves and transverse wind:

$$
\left(J_{\varphi \varphi}+\Delta J_{\varphi \varphi}\right) \ddot{\varphi}+2 N_{1} \dot{\varphi}+N_{3} \dot{\varphi}^{3}+C_{1} \varphi+C_{3} \varphi^{3}+C_{5} \varphi^{5}=F \cos w t+F_{\text {wind }}
$$

Wave force amplitude $F$ is usually expressed as $F=M g h . \alpha_{e 0}$ [44], where $h$ is transverse metacentric height, $\alpha_{e 0}$ is the effective wave slope angle, which is expressed as $\alpha_{e 0}=X_{\varphi} \alpha_{0}$, $X_{\varphi}$ is the effective wave slope angle correction which consider the influence of ship breadth and draught limitation [45]. The wave slope angle $\alpha_{0}=k \zeta_{a}, \quad k$ is the wave number, $\zeta_{a}$ is the wave amplitude.

\subsubsection{Symmetric system with no wind}

Firstly, we analyze the situation when there is no wind, for the roll motion equation (10),

Set $d_{1}=2 v_{\varphi \varphi}, d_{3}=v_{3}$, then equation (10) becomes:

$$
\ddot{\varphi}+d_{1} \dot{\varphi}+d_{3} \dot{\varphi}^{3}+r_{1} \varphi+r_{3} \varphi^{3}+r_{5} \varphi^{5}=f \cos w t
$$


Yihan Zhang, Ping Wang, Yachong Liu, Jingfeng Hu.
Nonlinear rolling stability and chaos research of trimaran vessel with variable lay-outs in regular and irregular waves under wind load

Set $x=\varphi, y=\dot{\varphi}$, then,

$$
\left\{\begin{array}{c}
\dot{x}=y \\
\dot{y}=-d_{1} y-d_{3} y^{3}-\left(r_{1} x+r_{3} x^{3}+r_{5} x^{5}\right)+f \cos w t
\end{array}\right.
$$

According to the nonlinear dynamic method, the small parameter $\varepsilon$ is used for the external force, so,

and equation (13) turns into:

$$
d_{1}=\varepsilon \bar{d}_{1}, d_{3}=\varepsilon \bar{d}_{3}, f=\varepsilon \bar{f}
$$

$$
\left\{\begin{array}{c}
\dot{x}=y \\
\dot{y}=-\varepsilon\left(\bar{d}_{1} y+\bar{d}_{3} y^{3}\right)-\left(r_{1} x+r_{3} x^{3}+r_{5} x^{5}\right)+\varepsilon \bar{f} \cos w t
\end{array}\right.
$$

When $\varepsilon=0$, the system degrades to a non-interference autonomous system,

$$
\left\{\begin{array}{c}
\dot{x}=y \\
\dot{y}=-\left(r_{1} x+r_{3} x^{3}+r_{5} x^{5}\right)
\end{array}\right.
$$

From the perspective of energy, the restoring moment corresponds to the potential energy, which is expressed as follows,

$$
V(x)=\frac{1}{2} r_{1} x^{2}+\frac{1}{4} r_{3} x^{4}+\frac{1}{6} r_{5} x^{6}
$$

For a system without damping and external excitation, it can be regarded as a Hamilton system. In order to solve the homoclinic or heteroclinic orbits, the Hamilton quantity $H(x)$ is used.

$$
H(x)=\frac{1}{2} y^{2}+\frac{1}{2} r_{1} x^{2}+\frac{1}{4} r_{3} x^{4}+\frac{1}{6} r_{5} x^{6}
$$

According to the initial condition, $\dot{x}=0, \dot{y}=0$, five equilibrium points can be calculated for the system (16),

$$
(0,0),\left( \pm \sqrt{\frac{\sqrt{r_{3}^{2}-4 r_{1} r_{5}}-r_{3}}{2 r_{5}}}, 0\right),\left( \pm \sqrt{\frac{-\sqrt{r_{3}^{2}-4 r_{1} r_{5}}-r_{3}}{2 r_{5}}}, 0\right)
$$

The characteristics of five equilibrium points are closely related to the stability of the system. The characteristic equation of system (16) is shown in equation (20).

$$
\left|\begin{array}{cc}
-\lambda & 1 \\
-r_{1}-3 r_{3} x^{2}-5 r_{5} x^{4} & -\lambda
\end{array}\right|=0
$$

$\lambda_{1,2}$ are characteristic values.

$$
\lambda_{1,2}= \pm \sqrt{-r_{1}-3 r_{3} x^{2}-5 r_{5} x^{4}}
$$

We calculated the equilibrium points and eigenvalues of all layouts, as shown in table 3 , $\lambda_{1,2}$ are the characteristic values corresponding to $x_{2}, x_{3}$, and $\lambda_{3,4}$ are the characteristic values corresponding to $x_{4}, x_{5}$.

$x_{2}, x_{3}, x_{4}, x_{5}$ respectively correspond to the following four equilibrium points.

$$
\left(\sqrt{\frac{\sqrt{r_{3}^{2}-4 r_{1} r_{5}}-r_{3}}{2 r_{5}}}, 0\right),\left(-\sqrt{\frac{\sqrt{r_{3}^{2}-4 r_{1} r_{5}}-r_{3}}{2 r_{5}}}, 0\right)\left(\sqrt{\frac{-\sqrt{r_{3}^{2}-4 r_{1} r_{5}}-r_{3}}{2 r_{5}}}, 0\right),\left(-\sqrt{\frac{-\sqrt{r_{3}^{2}-4 r_{1} r_{5}}-r_{3}}{2 r_{5}}}, 0\right)
$$

Fig.10 shows the phase portraits of the non-interference autonomous system at different CLs. In general, for the rolling system of the trimaran vessel, according to the classification method of homoclinic and heteroclinic orbits [14], stable attractor domain consists of two heteroclinic orbits $\Gamma_{1}$ and $\Gamma_{2}$, shown in Fig.10-(a). 
Table 3 Equilibrium points and characteristic roots at different CL

\begin{tabular}{|c|c|c|c|c|c|c|c|c|}
\hline & $\mathrm{CL}=4.5 \mathrm{~m}$ & $\mathrm{CL}=4.8 \mathrm{~m}$ & $\mathrm{CL}=5.0 \mathrm{~m}$ & $\mathrm{CL}=5.5 \mathrm{~m}$ & $\mathrm{CL}=6.0 \mathrm{~m}$ & $\mathrm{CL}=6.5 \mathrm{~m}$ & $\mathrm{CL}=7.0 \mathrm{~m}$ & $\mathrm{CL} \geq 8.0 \mathrm{~m}$ \\
\hline$x_{2}$ & 1.20 & 1.30 & 1.34 & 1.40 & 1.47 & 1.49 & 1.53 & none \\
\hline$x_{3}$ & -1.20 & -1.30 & -1.34 & -1.40 & -1.47 & -1.49 & -1.53 & none \\
\hline$x_{4}$ & 6.57 & 4.08 & 3.02 & 2.30 & 2.0 & 1.88 & 1.77 & none \\
\hline$x_{5}$ & -6.57 & -4.08 & -3.02 & -2.30 & -2.0 & -1.88 & -1.77 & none \\
\hline$\lambda_{1,2}$ & \pm 0.6 & \pm 0.7 & \pm 0.75 & \pm 0.82 & \pm 0.8 & \pm 0.78 & \pm 0.69 & none \\
\hline$\lambda_{3,4}$ & $\pm 3.3 \mathrm{i}$ & $\pm 2.2 \mathrm{i}$ & $\pm 1.7 \mathrm{i}$ & $\pm 1.3 \mathrm{i}$ & $\pm 1.08 \mathrm{i}$ & $\pm 0.98 \mathrm{i}$ & $\pm 0.79 \mathrm{i}$ & none \\
\hline
\end{tabular}

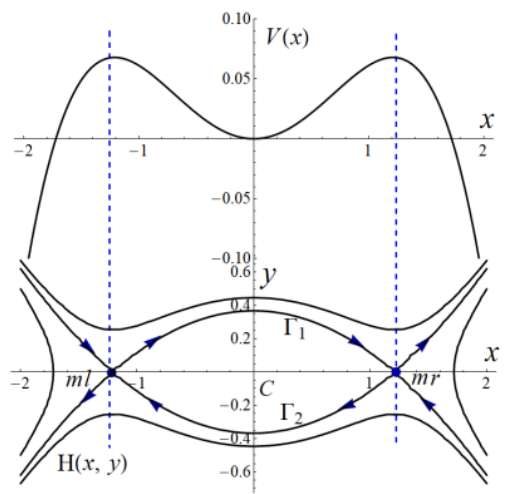

(a) $\mathrm{CL}=4.5 \mathrm{~m}$

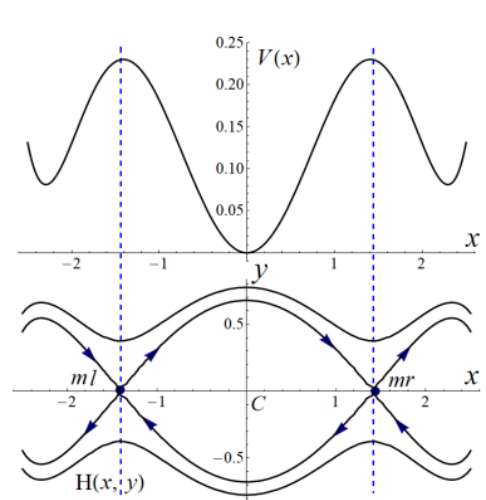

(d) $\mathrm{CL}=5.5 \mathrm{~m}$

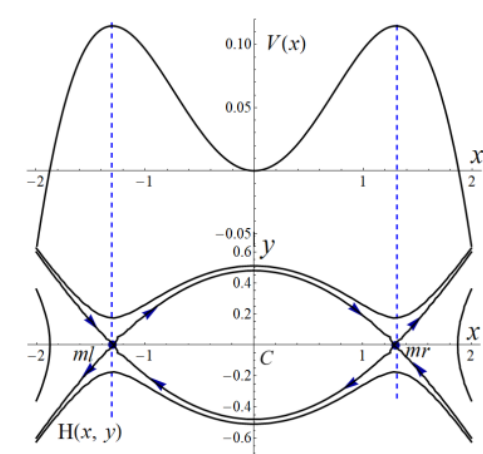

(b) $\mathrm{CL}=4.8 \mathrm{~m}$

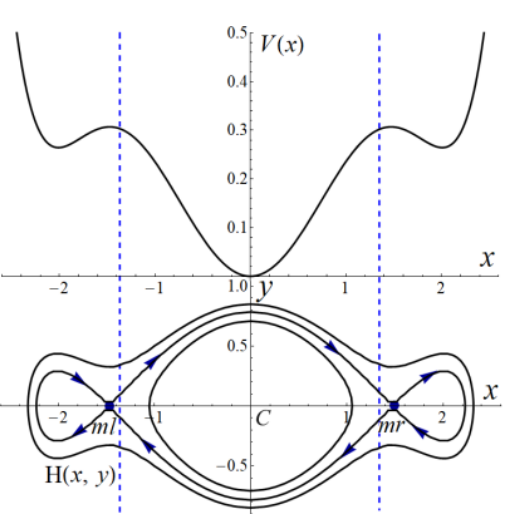

(e) $\mathrm{CL}=6.0 \mathrm{~m}$

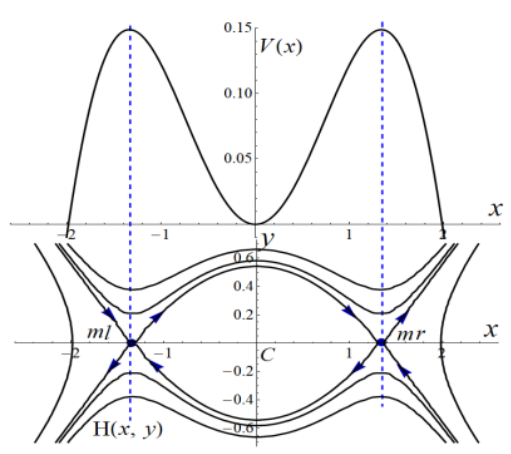

(c) $\mathrm{CL}=5.0 \mathrm{~m}$

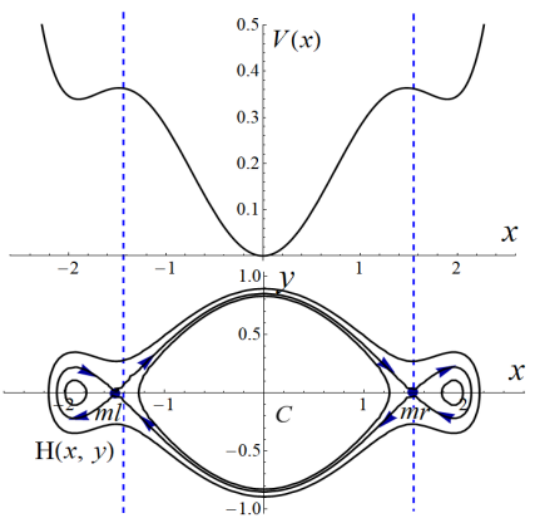

(f) $\mathrm{CL}=6.5 \mathrm{~m}$

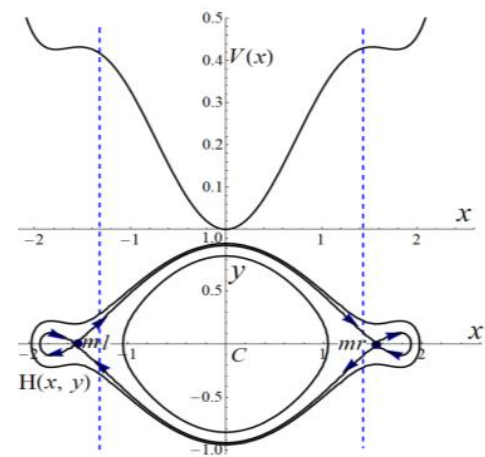

(g) $\mathrm{CL}=7.0 \mathrm{~m}$

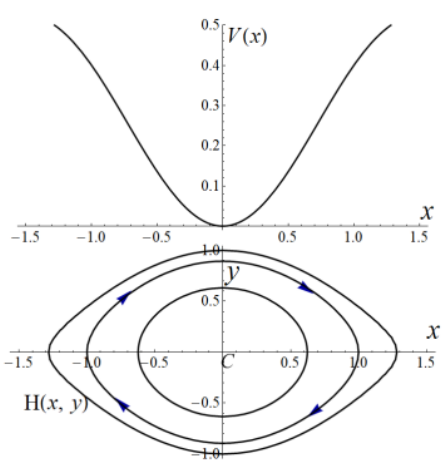

(h) $\mathrm{CL}=8.0 \mathrm{~m}$

Fig.10 Phase portraits of rolling Hamilton systems at different CL

The characteristic value of $x_{1}=(0,0)$ is a pure virtual root; therefore, it is impossible to determine its properties according to the linearization method. However, the Hamiltonian, as Lyapunov function, is greater than zero in the vicinity of $x_{1}=(0,0)$, so, $x_{1}=(0,0)$ can be 
Yihan Zhang, Ping Wang, Yachong Liu, Jingfeng $\mathrm{Hu}$.
Nonlinear rolling stability and chaos research of trimaran vessel with variable lay-outs in regular and irregular waves under wind load

judged as the center point which is at stable equilibrium state. When the transverse space CL is less than $8 \mathrm{~m}$, the characteristic values corresponding to $x_{2}, x_{3}$ are real numbers, and it can be determined that the equilibrium points corresponding to $x_{2}, x_{3}$ are the saddle points where two heteroclinic orbits intersect, which is exactly the vanishing angle of the ship's stability. The characteristic value of $x_{4}, x_{5}$ are pure imaginary roots, similar to $x_{1}$, the corresponding type of equilibrium points are also the central point, which are the unstable attractors for ship roll motion. The left orbit passing through the left saddle point and the right orbit passing through the right saddle point are homoclinic orbits, as shown in Fig.10 However, the homoclinic orbits has exceeded the motion bounds $(x=\pi)$, in reality, ship roll motion loses its practical significance, therefore, it has no significance for discussion.

It can also be seen from Fig. 10 and Table 3 that, with the increase of the transverse spacing, the abscissa of the saddle point turns away from the center point $\mathrm{C}$, and the attractor area of the intersection of the two heteroclinic rails becomes larger and larger. In a physical sense, that is, the larger the stability range of the corresponding Hamilton system, and the vanishing angle of stability is infinitely close to 90 degrees, leading to more stable state for the trimaran vessel. On the other hand, with the increase of CL, the position of the saddle point is further and further away from $x_{1}=(0,0)$, while the positions of the other two unstable center points are closer and closer to $x_{1}=(0,0), x_{2}, x_{3}$ and $x_{4}, x_{5}$ get closer, and finally disappears. The attractor areas of $x_{4}$ and $x_{5}$ bounded by homoclinic orbits is shrinking and finally disappearing as the saddle point disappears. When CL $\geq 8 \mathrm{~m}$, the system is left with only one central point, which is in a globally stable stat. Physically, when other conditions of the trimaran vessel remain unchanged, and the transverse spacing CL reaches a certain distance, the vanishing Angle of stability will not exist in the quadrants of $0 \sim \frac{\pi}{2}$, no matter how much angle it turns, it can be restored to the original state. Looking at the potential function, a potential well can be found both at the center point and the saddle point. As the CL increases, the potential energy generally tends to increase in the phase plane of roll motion.

\subsubsection{Asymmetric system with wind}

When the wind speed is constant, the wind load action to the trimaran is related to the heeling angle, which is similar to the restoring force. So, the wind load can be taken as a part of the undisturbed Hamilton system, and the nonlinear rolling motion system of the trimaran can be expressed as:

$\left\{\begin{array}{c}\dot{x}=y \\ \dot{y}=f_{\text {wind } 0}\left(1-\frac{x^{2}}{2}+\frac{x^{4}}{24}\right)-\varepsilon\left(\bar{d}_{1} y+\bar{d}_{3} y^{3}\right)-\left(r_{1} x+r_{3} x^{3}+r_{5} x^{5}\right)+\varepsilon \bar{f} \cos w t\end{array}\right.$

When, $\varepsilon=0$, rolling system become the undisturbed Hamilton system.

$$
\left\{\begin{array}{c}
\dot{x}=y \\
\dot{y}=f_{\text {wind } 0}\left(1-\frac{x^{2}}{2}+\frac{x^{4}}{24}\right)-\left(r_{1} x+r_{3} x^{3}+r_{5} x^{5}\right)
\end{array}\right.
$$

The potential energy equation is shown as below,

$$
V(x)=\frac{1}{2} r_{1} x^{2}+\frac{1}{4} r_{3} x^{4}+\frac{1}{6} r_{5} x^{6}-f_{\text {wind } 0}\left(1-\frac{x^{3}}{6}+\frac{x^{5}}{120}\right)
$$

It is similar to the state of no wind, where Hamilton quantity is:

$$
H(x)=\frac{1}{2} y^{2}+\frac{1}{2} r_{1} x^{2}+\frac{1}{4} r_{3} x^{4}+\frac{1}{6} r_{5} x^{6}-f_{\text {wind } 0}\left(1-\frac{x^{3}}{6}+\frac{x^{5}}{120}\right)
$$

The characteristic equation of the undisturbed autonomous system is used to solve the equilibrium point, and the Hamiltonian of the equilibrium point is used to solve the homoclinic or heteroclinic orbits, which forms the phase plane of the rolling motion. For the trimaran ships 
with different transverse spacing, the wind speed are $0 \mathrm{~m} / \mathrm{s}, 20 \mathrm{~m} / \mathrm{s}, 30 \mathrm{~m} / \mathrm{s}$ and $40 \mathrm{~m} / \mathrm{s}$. Fig. 11 shows the phase plane and potential energy function when $C L=4.5 \mathrm{~m}$ under different wind speed. When the wind load is not considered, the undisturbed system is a symmetric system, with a center point $\mathrm{C}$ and two saddle points $\mathrm{ml}$ and $\mathrm{mr}$. The saddle point is the intersection point of two heteroclinic orbits, and the potential function is symmetric about the Y-axis. With the increase of wind speed, the wind force moment gradually increases, and the symmetry of the system is destroyed. The central equilibrium point $\mathrm{C}$ of the system is shifted to the right, and the right saddle point develops into the intersection point of a homoclinic orbit. With the increase of wind speed, the safe basin surrounded by the homoclinic orbit becomes smaller, making the ship more vulnerable to loss of stability. It is also worth noting that the potential energy function is also asymmetrical, with the potential energy on the right plane decreasing and that on the left plane increasing.

Fig.12 shows the phase plane and potential energy function of trimaran at different transverse spacing. It can be intuitively found that, with the increase of spacing, the center point moves to the origin, and the coordinates of the saddle point and the area of the safe basin increases gradually, indicating that nonlinear system become more stable. The asymmetry of the potential function is decreasing. For the unstable left saddle point $\mathrm{ml}$, the intersection point of the homoclinic orbit gradually develops to the homoclinic orbit. It is worth noting that when $\mathrm{CL}=8$, the saddle point disappears and the system has only one central equilibrium point. For the ship moving plane $x \in[-1.57,1.57]$, the state of global stability will be reached.

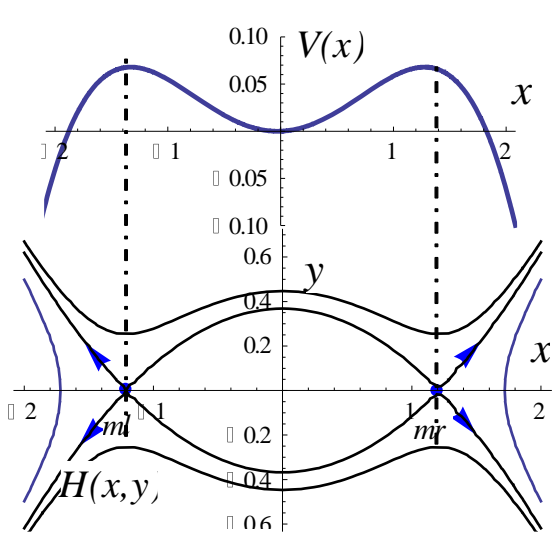

(a) $\mathrm{CL}=4.5 \mathrm{~m}$, Vwind $=0 \mathrm{~m} / \mathrm{s}$

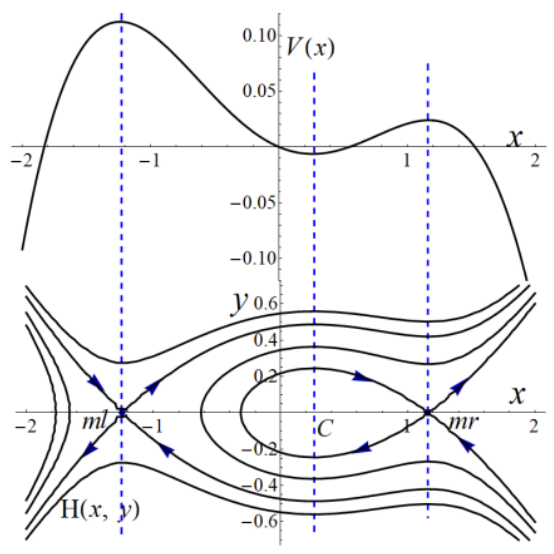

(c) $C L=4.5 \mathrm{~m}, \mathrm{~V}$ wind $=20 \mathrm{~m} / \mathrm{s}$

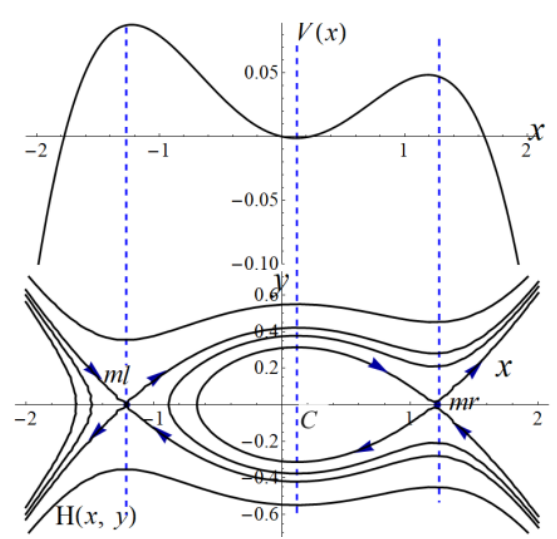

(b) $\mathrm{CL}=4.5 \mathrm{~m}, \mathrm{~V}$ wind $=10 \mathrm{~m} / \mathrm{s}$

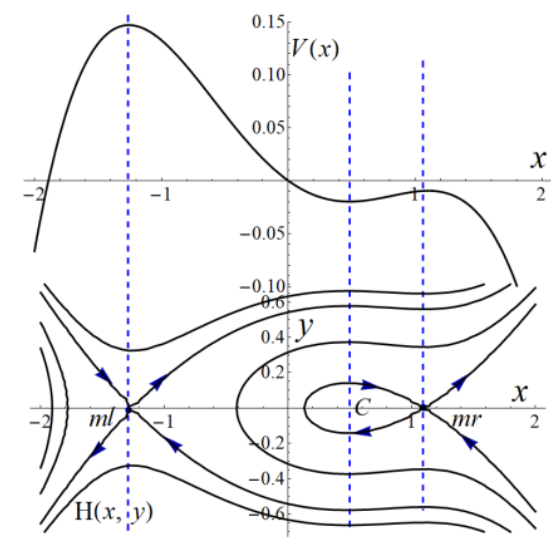

(d) $\mathrm{CL}=4.5 \mathrm{~m}, \mathrm{~V}$ wind $=30 \mathrm{~m} / \mathrm{s}$

Fig. 11 Phase portrait of $\mathrm{cl}=4.5 \mathrm{~m}$ under different wind speed 
Yihan Zhang, Ping Wang, Yachong Liu, Jingfeng $\mathrm{Hu}$.
Nonlinear rolling stability and chaos research of trimaran vessel with variable lay-outs in regular and irregular waves under wind load

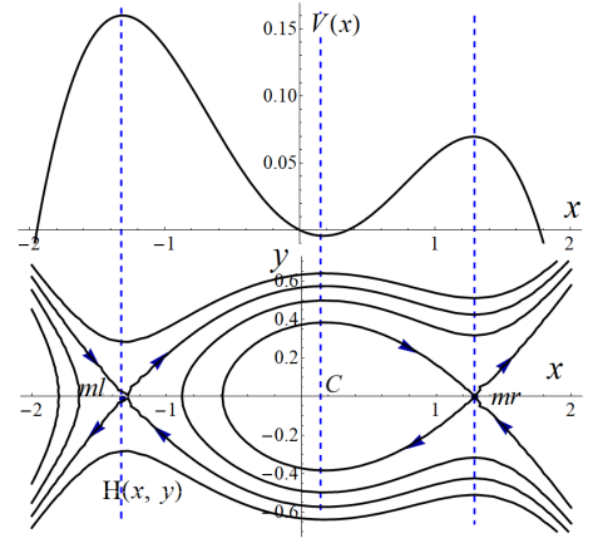

(a) $\mathrm{CL}=4.8 \mathrm{~m}, \mathrm{~V}$ wind $=20 \mathrm{~m} / \mathrm{s}$

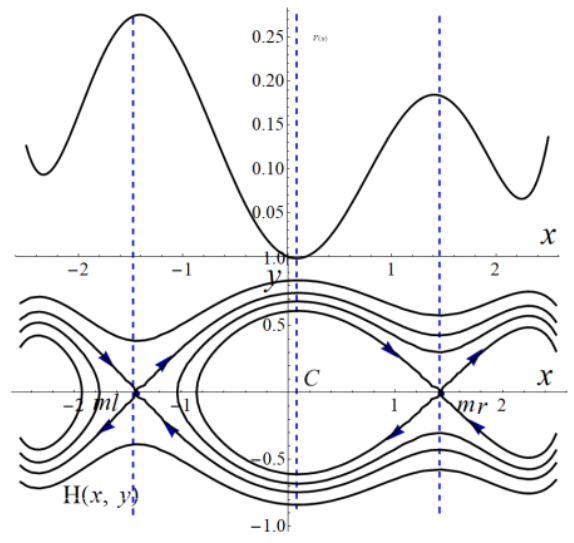

(c) $\mathrm{CL}=5.5 \mathrm{~m}, \mathrm{Vwind}=20 \mathrm{~m} / \mathrm{s}$

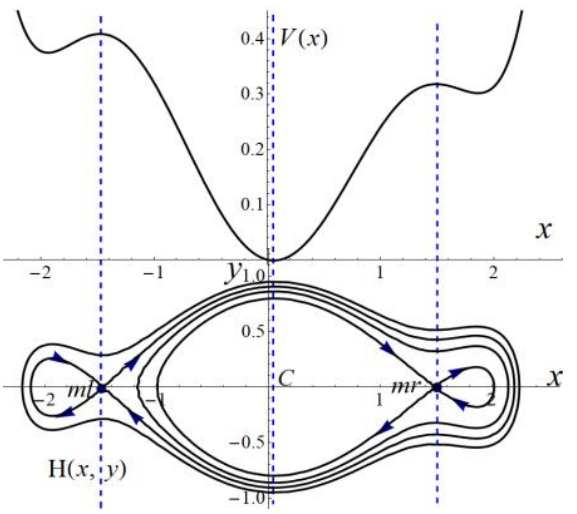

(e) $\mathrm{CL}=6.5 \mathrm{~m}$, Vwind $=20 \mathrm{~m} / \mathrm{s}$

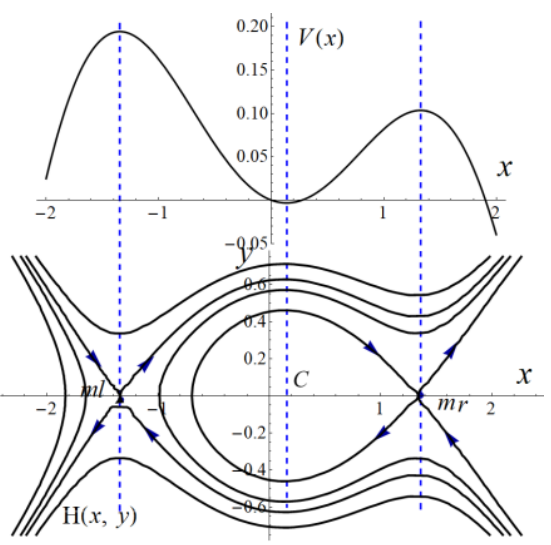

(b) $\mathrm{CL}=5.0 \mathrm{~m}, \mathrm{~V}$ wind $=20 \mathrm{~m} / \mathrm{s}$

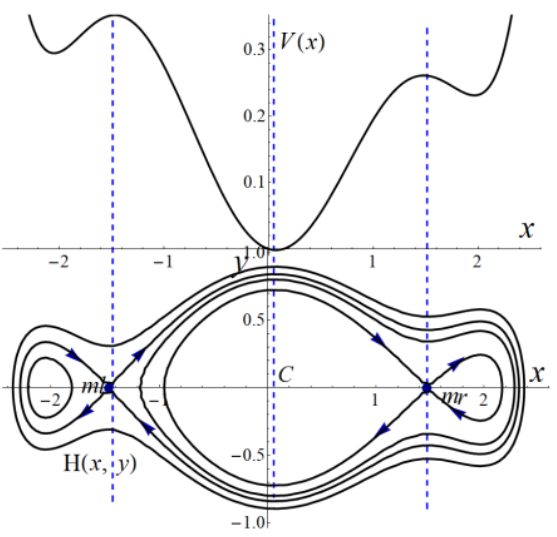

(d) $\mathrm{CL}=6.0 \mathrm{~m}$, Vwind $=20 \mathrm{~m} / \mathrm{s}$

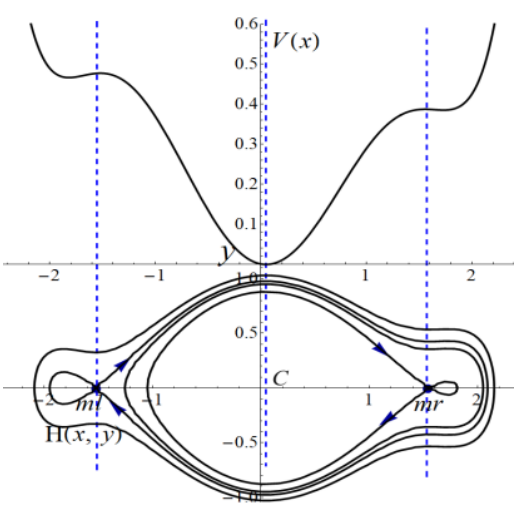

(f) $\mathrm{CL}=7.0 \mathrm{~m}, \mathrm{~V}$ wind $=20 \mathrm{~m} / \mathrm{s}$

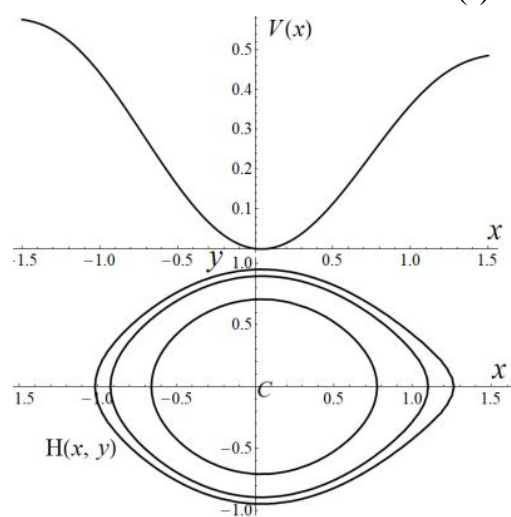

(f) $\mathrm{CL}=8.0 \mathrm{~m}, \mathrm{Vwind}=20 \mathrm{~m} / \mathrm{s}$

Fig. 12 Phase portrait of different $\mathrm{cl}$ under wind speed of $20 \mathrm{~m} / \mathrm{s}$ 


\subsection{Melnikov function}

Melnikov function is a typical analytic method to solve the external excitation threshold of a chaotic system, although it returns the conservative side results in some engineering applications. According to the theory of Melnikov's function, when the non-autonomous system connects the unstable shape flow and stable shape flow of homoclinic or heteroclinic saddle points, it can be determined that the system has chaos in the sense of Smale horseshoe transformation [14]. The meaning of Melnikov function is the distance between stable form flow and unstable form flow, which is always applied to calculate the threshold of external excitation when the system is entering the chaotic state.

The two-dimensional non-autonomous system can be expressed as $\dot{x}=f(x)+\varepsilon g(x, t)$.

For nonlinear roll motion model of the trimaran vessel, there is only heteroclinic orbits in the real motion plane as discussed above. The distance between stable and unstable form flow through saddle point $x=m 0$ is expressed as:

$$
M\left(t_{0}\right)=\int_{-\infty}^{+\infty} f\left(\bar{x}_{0}(t)\right) \wedge \mathrm{g}\left(\bar{x}_{0}(t), t+t_{0}\right) \exp \left[-\int_{-\infty}^{+\infty} \operatorname{trace}\left(D f\left(\bar{x}_{0}(s)\right)\right) d s\right] d t
$$

Where $\bar{x}_{0}(t)$ is the heteroclinic orbital equation through $m 0$, and $\operatorname{trace}\left(D f\left(\bar{x}_{0}(s)\right)\right)$ is the trace of the matrix.

For rolling autonomous undisturbed system, $\operatorname{trace}\left(D f\left(\bar{x}_{0}(s)\right)\right) \equiv 0$,

So, the Melnikov function is simplified to:

$$
M\left(t_{0}\right)=\int_{-\infty}^{+\infty} f\left(\bar{x}_{0}(t)\right) \wedge \mathrm{g}\left(\bar{x}_{0}(t), t+t_{0}\right) d t
$$

For the rolling system (22), Melnikov function can be obtained as follows.

$$
M\left(t_{0}\right)=\int_{-\infty}^{\infty} \bar{f} \cos \left(w t_{0}\right) y \cos (\omega t) d t-\int_{-\infty}^{\infty} \bar{f} \sin \left(w t_{0}\right) y \sin (\omega t) d t-\bar{d}_{1} \int_{0}^{\infty} y^{2} d t-
$$

By further simplification,

$$
M\left(t_{0}\right)=\bar{f} \cos \left(w t_{0}\right) I_{1}-\bar{f} \sin \left(w t_{0}\right) I_{2}-\bar{d}_{1} I_{3}-\bar{d}_{3} I_{4}
$$

Where, $\quad I_{1}=\int_{m r l}^{m r r} \cos (\omega t) d x \quad, \quad I_{2}=\int_{m r l}^{m r r} \sin (\omega t) d x \quad, \quad I_{3}=\int_{m r l}^{m r r} y d x \quad, \quad I_{4}=$ $\int_{m r l}^{m r r} y^{3} d x, m r l, \quad m r r$ are the $x$ coordinates of the left and right boundaries of safe basin in the phase plane. The above integrals are solved by numerical integration method of GaussLegendre. When $M\left(t_{0}\right)=0$, Melnikov function appears simple zero root, and the system loses stability due to chaos.

$$
\bar{f}_{t}=\left|\bar{d}_{1} I_{3}+\bar{d}_{3} I_{4}\right| / \sqrt{I_{1}^{2}+I_{2}^{2}}
$$

Multiply $\varepsilon$ for both sides of this equation, the critical value of external excitation for the real ship is

$$
f_{t}=\left|d_{1} I_{3}+d_{3} I_{4}\right| / \sqrt{I_{1}^{2}+I_{2}^{2}}
$$

For the rolling system, $f_{t}$ is the threshold of wave force when chaos happens.

According to the quantity of Hamilton,

$$
y= \pm \sqrt{2 H(x, y)-\left(r_{1} x^{2}+r_{3} x^{4} / 2+r_{5} x^{6} / 3\right)+f_{\text {wind } 0}\left(x-\frac{x^{3}}{3}+\frac{x^{5}}{60}\right)}
$$

Because the above equation involves the $x^{6}$ term, it is impossible to get the analytic expression of heteroclinic orbit, which usually needs to be solved by numerical integration.

From $y=\dot{x}=d x / d t$, after integrating both sides, 
Yihan Zhang, Ping Wang, Yachong Liu, Jingfeng $\mathrm{Hu}$.
Nonlinear rolling stability and chaos research of trimaran vessel with variable lay-outs in regular and irregular waves under wind load

$$
t= \pm \int_{0}^{x} \frac{1}{\sqrt{2 H\left(x_{m}, y_{m}\right)-\left(r_{1} \varsigma^{2}+r_{3} \varsigma^{4} / 2+r_{5} \varsigma^{6} / 3\right)+f_{\text {wind } 0}\left(\varsigma-\frac{\varsigma^{3}}{3}+\frac{\varsigma^{5}}{600}\right)}} d \varsigma
$$

The three points integral formula of Gauss-Legendre integral is used to calculate the integral $I_{1}, I_{2}, I_{3}, I_{4}$, and the gaussian points and weight coefficients can refer to the numerical integration textbook.

The above method is applied to calculate the wave force threshold under different wave frequencies for the trimaran with different transverse spacing. As for the trimaran itself, with the increase of wave excitation frequency, the threshold also increases. Fig.13 shows the wave threshold with no wind force. From the perspective of layout variation, the threshold value generally increases with the increase of CL, which is identical to the results of phase portrait analysis of Hamilton system. However, considering the nonlinear roll damping, when the roll motion is violent, nonlinear roll damping will produce great effect to the roll system, which can be found from table 2, when spacing increasing after $\mathrm{CL}=5.5 \mathrm{~m}$, nonlinear damping shows the tendency of decrease, so for $\mathrm{CL}=6.0 \mathrm{~m}, \mathrm{CL}=6.5 \mathrm{~m}$ and $\mathrm{CL}=7.0 \mathrm{~m}$, their threshold values are less than some small spacing, especially in the high frequency obviously. Theoretically, when the wave force excitation exceeds the threshold, chaos will occur in the system, causing unstable roll motion and capsizing.

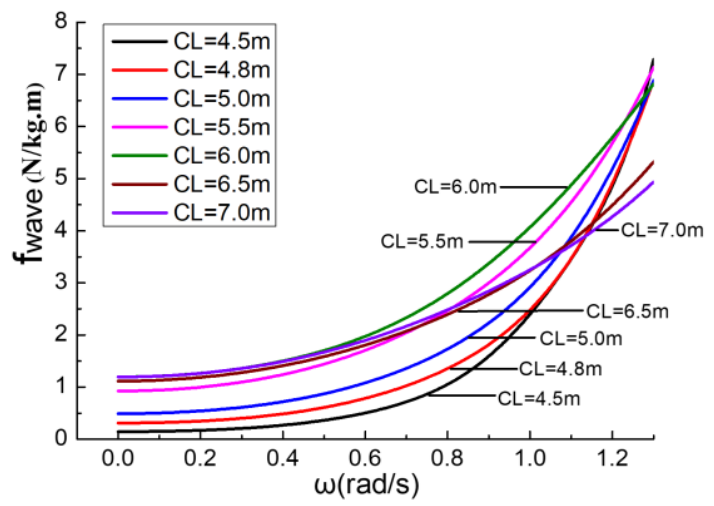

Fig.13 Wave force threshold at different CLs of the trimaran vessel (Vwind $=0 \mathrm{~m} / \mathrm{s})$

Fig. 14 shows the threshold values of the distance $\mathrm{CL}=4.5 \mathrm{~m}$ for different wind speeds. It can be seen that the wave threshold gradually decreases with the increase of wind speed, which is consistent with the physical phenomenon. Fig. 15 shows the wave threshold values at different CL. With the increase of CL, the system stability is enhanced, which is consistent with the conclusion of phase portrait analysis.

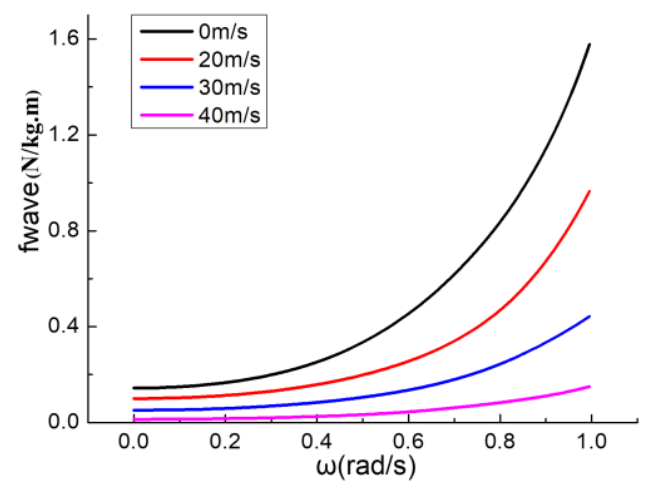

Fig.14. Wave force threshold under varying wind speed $(\mathrm{CL}=4.5 \mathrm{~m})$

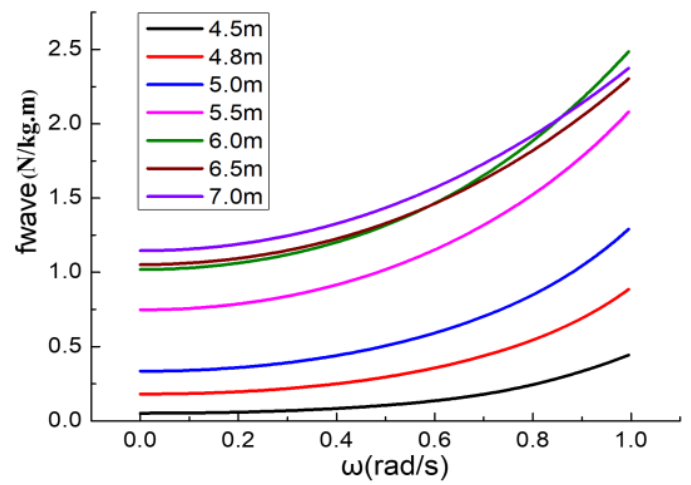

Fig.15. Wave force threshold of varying CL (Vwind $=30 \mathrm{~m} / \mathrm{s}$ ) 


\subsection{Numerical Verification}

The threshold of chaos can be calculated based on Melnikov function, but it is not a sufficient condition for chaos to occur in the system. In order to verify the accuracy of the wave force threshold for trimaran roll system, other methods are needed for numerical verification. In the field of nonlinear dynamics, there are some methods for numerical verification of chaos, including Lyapunov exponent, power spectrum, fractal dimension, etc. In this paper, Lyapunov exponent is used to judge the chaotic characteristics of a certain threshold, and the global stability of rolling system is studied by the erosion of safe basin.

\subsubsection{Lyapunov exponent}

To the equilibrium point, the stability can be judged by the real part of characteristic value of the system's Jacobi matrix or the characteristic exponent of the periodic motion perturbation equation. Lyapunov exponent is a generalization of characteristic value and characteristic exponent, and gives a measure of the average divergence or average convergence of any adjacent orbits of the system, which is the most reliable measure to judge chaos.

Lyapunov exponent is expressed as:

$$
\lambda_{k}=\lim _{t \rightarrow+\infty} \frac{\ln \left\|W_{k}(t)\right\|}{t}, \quad k=1,2, \ldots, n
$$

Arrange them from large to small, $\lambda_{1} \geq \lambda_{2} \geq \ldots \geq \lambda_{n}$.

If the maximum Lyapunov exponent is less than zero, the system is stable. If the maximum Lyapunov exponent is greater than zero, chaos will appear in the system. For the nonautonomous two-dimensional roll motion system, there are only two Lyapunov exponents. The most common solution of Lyapunov exponents is RHR algorithm [46].

For two dimensional non-autonomous systems as follows.

$\ddot{x}+f(x, \dot{x}, t)=g(t)$, Set $x=y_{1}, \dot{x}=y_{2}, t=y_{3}$, it changes to be a three-dimensional autonomous system.

$$
\left\{\begin{array}{c}
\dot{y}_{1}=y_{2} \\
\dot{y}_{2}=g\left(y_{3}\right)-f\left(y_{1}, y_{2}, y_{2}\right) \\
\dot{y}_{3}=1
\end{array}\right.
$$

Obviously, the two Lyapunov exponents of the two-dimensional non-autonomous system are the same as the first two Lyapunov exponents of the corresponding three-dimensional autonomous system.

Carry on the division to autonomous system, and $\dot{Y}(t)=J(t) Y(t), Y(0)=I_{3}$ is the identity matrix, $J(t)$ is the Jacobian matrix. According to $J(t)$, the third-row element of $Y(t)$ is known, and $\mathrm{Y}(\mathrm{t})$ is a non-singular matrix, so $\mathrm{QR}$ decomposition can be performed as follows.

$$
\left[\begin{array}{ccc}
Y_{11} & Y_{12} & Y_{13} \\
Y_{21} & Y_{22} & Y_{23} \\
0 & 0 & 1
\end{array}\right]=\left[\begin{array}{ccc}
Q_{11} & Q_{12} & 0 \\
Q_{21} & Q_{22} & 0 \\
0 & 0 & 1
\end{array}\right]\left[\begin{array}{ccc}
R_{11} & R_{12} & R_{13} \\
0 & R_{22} & R_{23} \\
0 & 0 & 1
\end{array}\right]
$$

Since $R_{33}=1$, the first two Lyapunov exponentials can be obtained only by solving the two-dimensional subsystem, which can also be divided as follows,

$$
\dot{\bar{Y}}(t)=\bar{J}(t) \bar{Y}(t), \bar{J}(t)=\left[\begin{array}{ll}
J_{11} & J_{12} \\
J_{21} & J_{21}
\end{array}\right], \bar{Y}(t)=\bar{Q}(t) \bar{R}(t),
$$

Combine the above equations,

$$
\bar{Q}^{T} \dot{\bar{Q}}+\dot{\bar{R}} \bar{R}^{-1}=\bar{Q}^{T} \bar{J} \bar{Q}
$$

Based on RHR algorithm , the orthogonal matrix $\bar{Q}(t)$ is transformed as follows. 
Yihan Zhang, Ping Wang, Yachong Liu, Jingfeng Hu.
Nonlinear rolling stability and chaos research of trimaran vessel with variable lay-outs in regular and irregular waves under wind load

$$
\bar{Q}(t)=\left[\begin{array}{cc}
\cos \theta(t) & \sin \theta(t) \\
-\sin \theta(t) & \cos \theta(t)
\end{array}\right], \bar{R}(t)=\left[\begin{array}{cc}
e^{\lambda_{1}(t)} & r_{11} \\
0 & e^{\lambda_{2}(t)}
\end{array}\right]
$$

Thus, the solution system of the Lyapunov exponent can be expressed as, ,

$$
\left\{\begin{array}{c}
\frac{d \sigma_{1}(t)}{d t}=-\frac{\partial f}{\partial y_{2}} \\
\frac{d \sigma_{2}(t)}{d t}=-\frac{\partial f}{\partial y_{2}} \cos 2 \theta-\left(1-\frac{\partial f}{\partial y_{1}}\right) \sin 2 \theta \\
\frac{d \theta(t)}{d t}=-\frac{\partial f}{2 \partial y_{2}} \sin 2 \theta+\sin ^{2} \theta+\frac{\partial f}{\partial y_{1}} \cos ^{2} \theta
\end{array}\right.
$$

Where,

$$
\left\{\begin{array}{l}
\lambda_{1}(t)=\left(\sigma_{1}(t)+\sigma_{2}(t)\right) / 2 \\
\lambda_{2}(t)=\left(\sigma_{1}(t)-\sigma_{2}(t)\right) / 2
\end{array}\right.
$$

The two Lyapunov exponents of two-dimensional non-autonomous system were further solved as:

$$
\lambda_{1}=\lim _{t \rightarrow+\infty} \frac{\lambda_{1}(t)}{t}, \lambda_{2}=\lim _{t \rightarrow+\infty} \frac{\lambda_{2}(t)}{t}
$$

The parameters of the roll motion system are substituted into the equations (39), we can get the final calculation system.

$$
\left\{\begin{array}{c}
\frac{d \sigma_{1}(t)}{d t}=-\left(d_{1}+3 d_{3} y^{2}\right) \\
\frac{d \sigma_{1}(t)}{d t}=\left(d_{1}+3 d_{3} y^{2}\right) \cos 2 \theta-\sin 2 \theta+\left[r_{1}+3 r_{3} x^{2}+5 r_{5} x^{4}+f_{\text {wind } 0}\left(x-\frac{x^{3}}{6}\right)\right] \sin 2 \theta \\
\frac{d \theta(t)}{d t}=-\frac{1}{2}\left(d_{1}+3 d_{3} y^{2}\right) \sin 2 \theta+\sin ^{2} \theta+\left[r_{1}+3 r_{3} x^{2}+5 r_{5} x^{4}+f_{\text {wind } 0}\left(x-\frac{x^{3}}{6}\right)\right] \cos ^{2} \theta
\end{array}\right.
$$

In this paper, the 4-order Runge-Kutta method is applied to solve the equations (22) and (42), and the Lyapunov exponent can be obtained by combining equations (40) and (41). For the rolling system of trimaran vessel with different transverse layouts, it is verified according to the wave force excitation threshold obtained by Melnikov function. Fig.16 and Fig.17 show the Lyapunov exponent of two CLs when the wave frequency is $0.4 \mathrm{rad} / \mathrm{s}$, and the $\mathrm{ft}$ refers to wave force threshold. For the selected three lay-outs, when the excitation value is greater than the threshold, the maximum Lyapunov exponent is greater than zero, and the system is in a chaotic state, however, when the excitation is less than the threshold, the maximum Lyapunov exponent is less than zero, and the system is in a stable state. This result effectively verifies the wave force threshold calculated by Melnikov function.

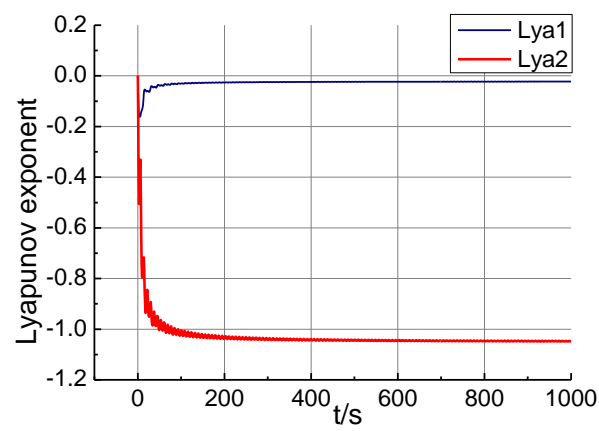

(a) $\mathrm{f}=0.26(\mathrm{ft}=0.27)$ Vwind $=0 \mathrm{~m} / \mathrm{s}$

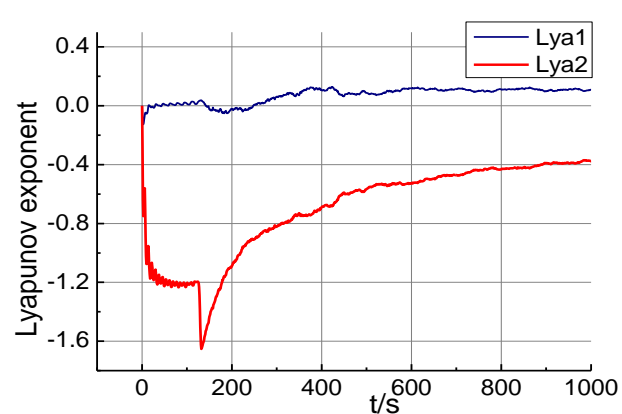

(b) $\mathrm{f}=0.28(\mathrm{ft}=0.27) \mathrm{V}$ wind $=0 \mathrm{~m} / \mathrm{s}$ 


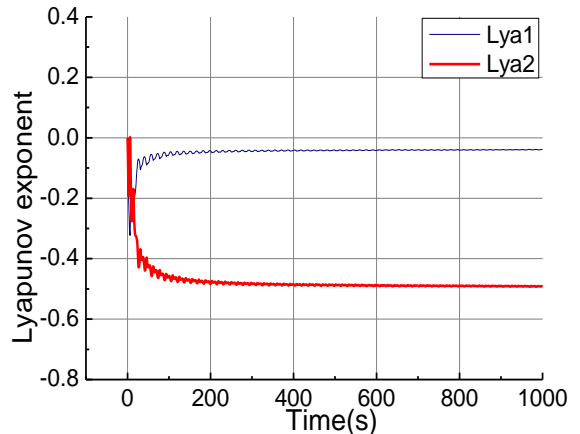

(c) fwave $=0.083(\mathrm{ft}=0.084)$ Vwind $=20 \mathrm{~m} / \mathrm{s}$

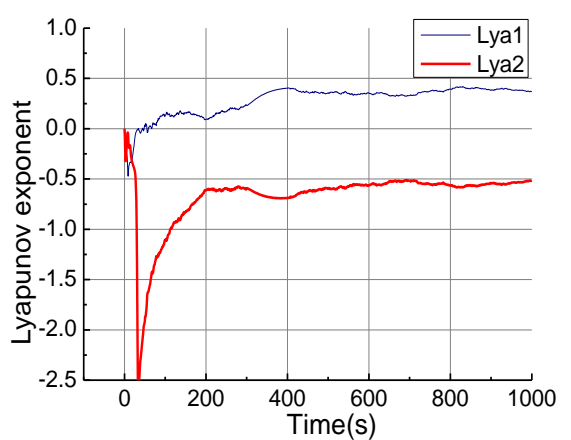

(d) fwave $=0.085(\mathrm{ft}=0.084)$ Vwind $=20 \mathrm{~m} / \mathrm{s}$

Fig.16 Lyapunov exponent at different wind speeds at $\mathrm{CL}=4.5 \mathrm{~m}$

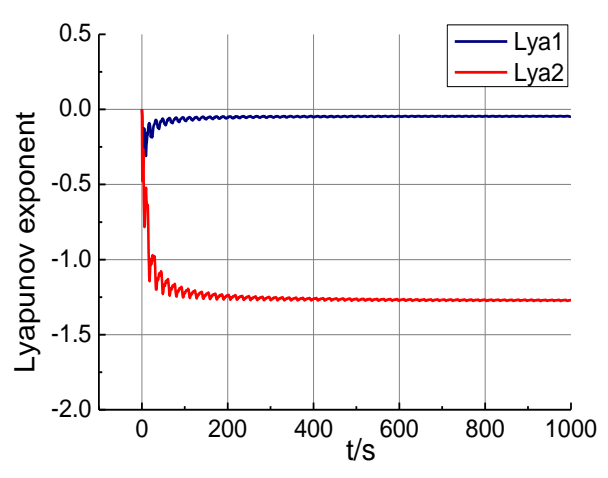

(a) $\mathrm{CL}=6.0 \mathrm{~m}, \mathrm{f}=1.4(\mathrm{ft}=1.5)$ Vwind $=0 \mathrm{~m} / \mathrm{s}$

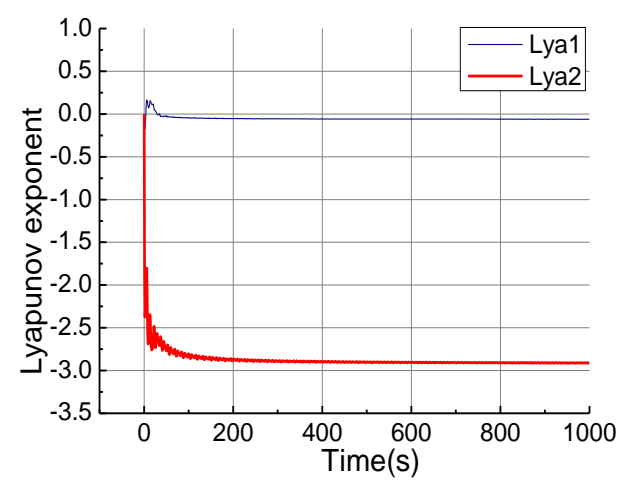

(c) fwave $=1.15(\mathrm{ft}=1.2)$ Vwind $=20 \mathrm{~m} / \mathrm{s}$

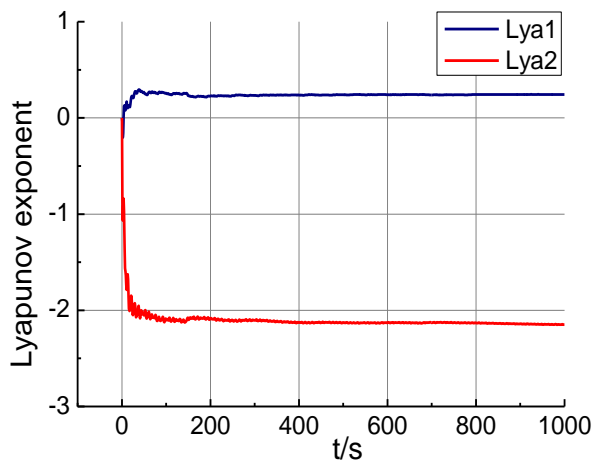

(b) $\mathrm{CL}=6.0 \mathrm{~m}, \mathrm{f}=1.6(\mathrm{ft}=1.5) \mathrm{V}$ wind $=0 \mathrm{~m} / \mathrm{s}$

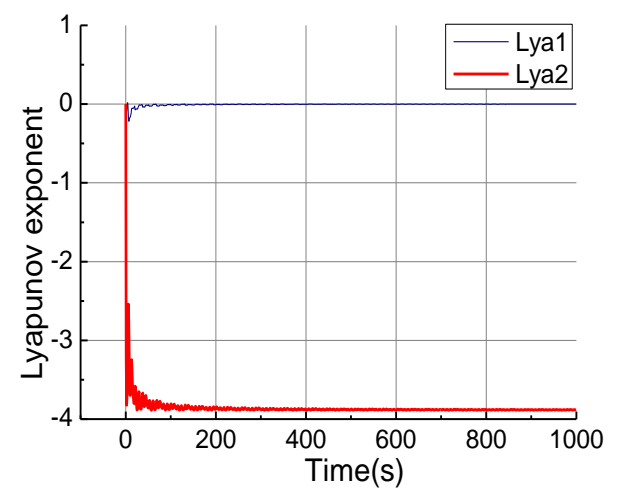

(d) fwave $=1.25(\mathrm{ft}=1.2)$ Vwind $=20 \mathrm{~m} / \mathrm{s}$

Fig.17 Lyapunov index at different wind speeds at CL $=6.0 \mathrm{~m}$

\subsubsection{Safe basin analysis}

For the roll motion system, the safety region can be defined as follows: on the phase plane, the numerical simulation of roll motion is carried out with different rolling angle and angular velocity as initial values. When the motion is not divergent, the rolling system is stable. By representing the distribution of the stable points and unstable points on the phase plane, the safe basin diagram of roll motion can be obtained [28, 47-48]. It can also be applied for the further verification of the wave force threshold obtained by Melnikov function. Take three kinds of transverse lay-outs for example, Fig. 18 show the safe basin of $C L=4.5 \mathrm{~m}, \mathrm{CL}=5.0 \mathrm{~m}$ and $\mathrm{CL}=6.0 \mathrm{~m}$, and keep the wave frequency as $0.4 \mathrm{rad} / \mathrm{s}$ as the same as Lyapunov calculation above. As we can see, when the external excitation increases from zero to the same threshold obtained from Melnikov method, the safe basin is relatively complete, and when the excitation exceeds the threshold, the stability domain begins to break, and chaos will be generated in the system. The wave force threshold obtained from Melnikov method is well validated by safe basin analysis. 
Yihan Zhang, Ping Wang, Yachong Liu, Jingfeng $\mathrm{Hu}$.
Nonlinear rolling stability and chaos research of trimaran vessel with variable lay-outs in regular and irregular waves under wind load

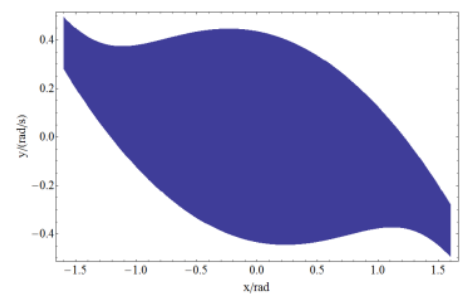

(a) $\mathrm{CL}=4.5 \mathrm{~m}, \mathrm{~F}=0$

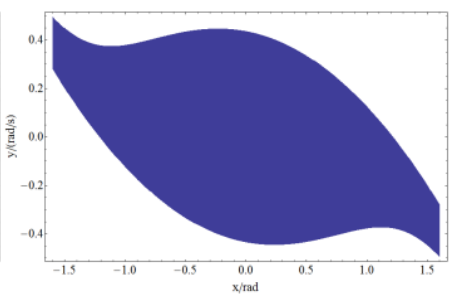

(b) $\mathrm{CL}=4.5 \mathrm{~m}, \mathrm{~F}=0.26$

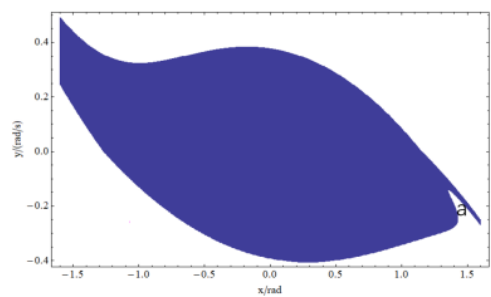

(c) $\mathrm{CL}=4.5 \mathrm{~m}, \mathrm{~F}=0.28$

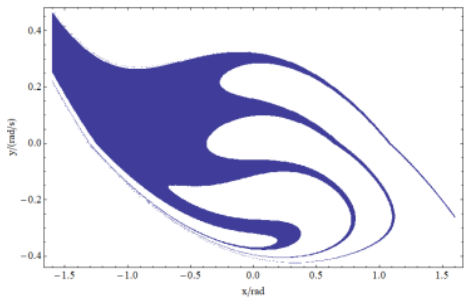

(d) $\mathrm{CL}=4.5 \mathrm{~m}, \mathrm{~F}=0.50$

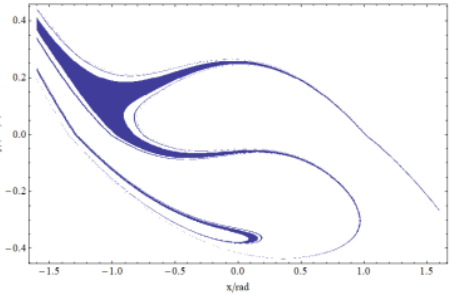

(e) $\mathrm{CL}=4.5 \mathrm{~m}, \mathrm{~F}=0.75$

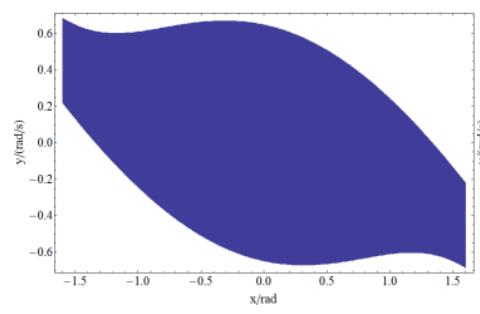

(f) $\mathrm{CL}=5.0 \mathrm{~m}, \mathrm{~F}=0$

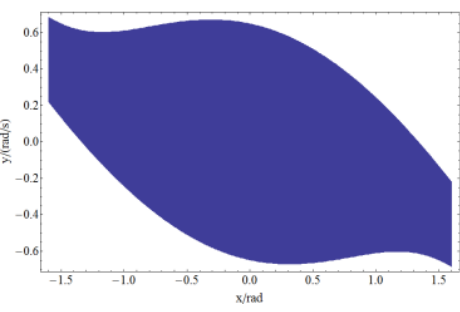

(g) $\mathrm{CL}=5.0 \mathrm{~m}, \mathrm{~F}=0.70$

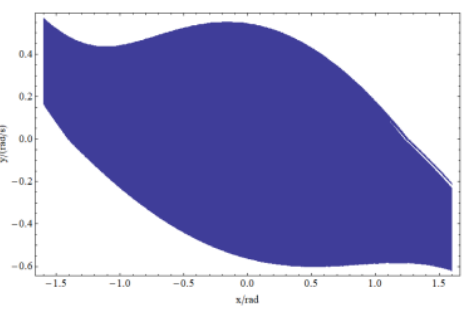

(h) $\mathrm{CL}=5.0 \mathrm{~m}, \mathrm{~F}=0.74$

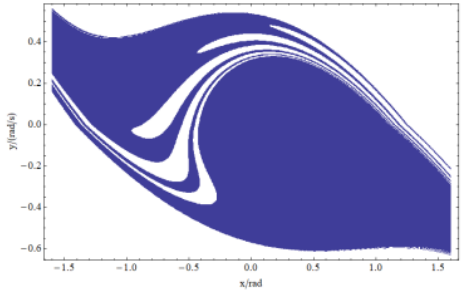

(j) $\mathrm{CL}=5.0 \mathrm{~m}, \mathrm{~F}=1.0$

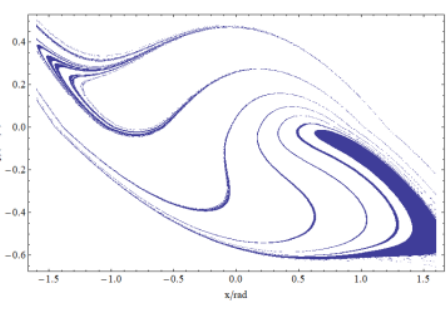

(j) $\mathrm{CL}=5.0 \mathrm{~m}, \mathrm{~F}=1.5$

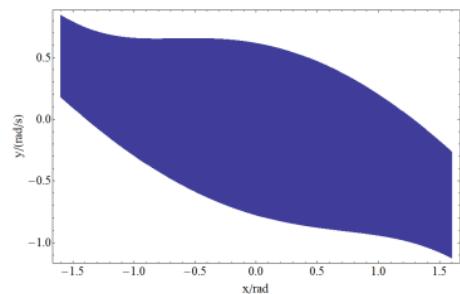

(i) $\mathrm{CL}=6.0 \mathrm{~m}, \mathrm{~F}=0$

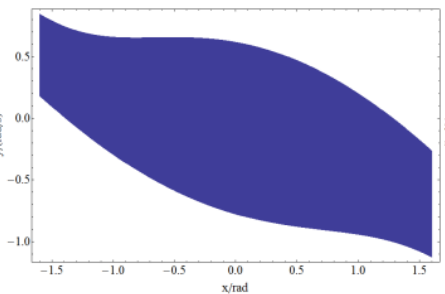

(j) $\mathrm{CL}=6.0 \mathrm{~m}, \mathrm{~F}=1.4$

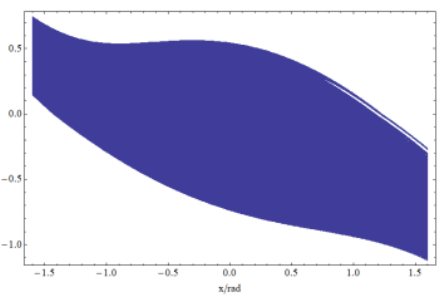

(k) $\mathrm{CL}=6.0 \mathrm{~m}, \mathrm{~F}=1.6$

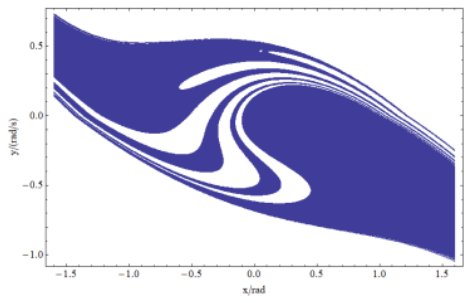

(1) $\mathrm{CL}=6.0 \mathrm{~m}, \mathrm{~F}=2.0$

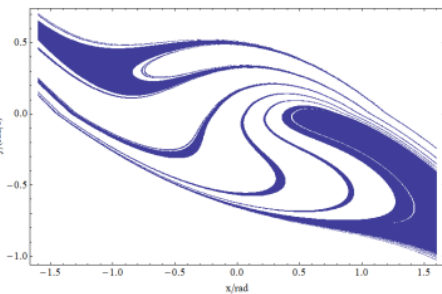

(m) $\mathrm{CL}=6.0 \mathrm{~m}, \mathrm{~F}=2.5$

Fig.18 The erosion of safe basin of trimaran at different CLs under the change of wave force when the wave frequency $\omega=0.4 \mathrm{rad} / \mathrm{s}$

Fig.19 shows the area of the safe basin. The beginning areas of the three CLs are different, and the larger the CL is, the larger the area will be, which is related to the restoring force coefficient [49]. However, with the increase of wave force excitation, when the threshold is 
reached, the area of safe basin starts to break. The trimaran vessel with small CL first begins to break down, and the rolling system becomes more unstable than the ship with big CL.

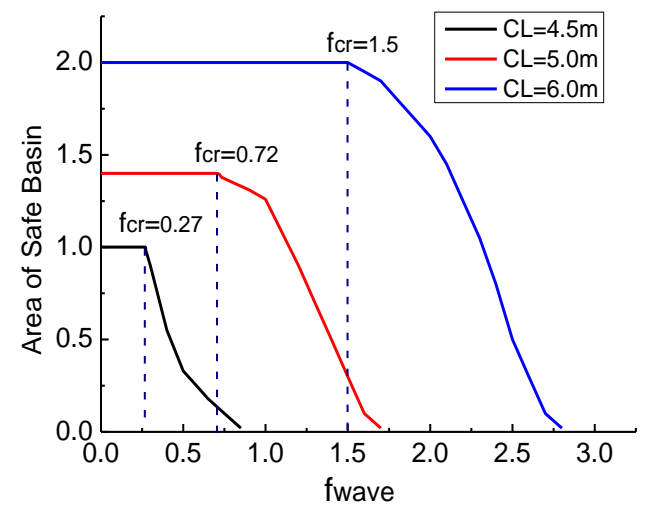

Fig.19 The change curves of the safe basin area

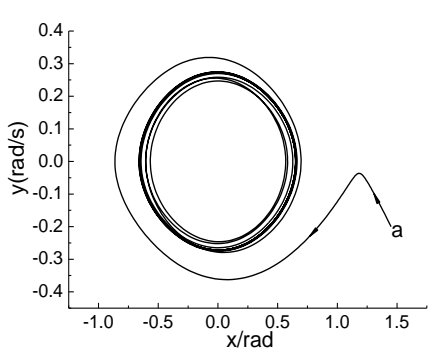

(a) $f=0.26$

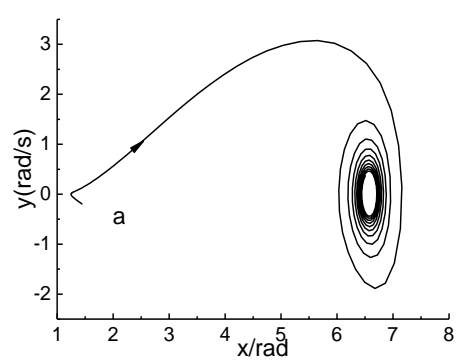

(b) $\mathrm{f}=0.28$

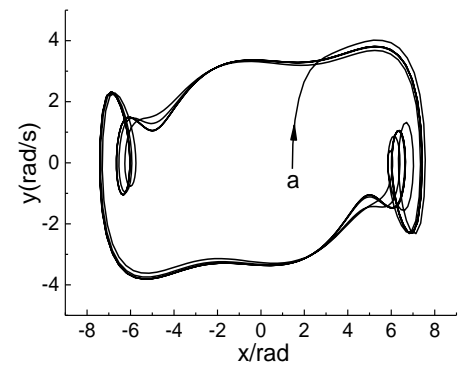

(c) $f=0.75$

Fig.20 Roll motion trails of point a $(1.45,-0.2)$ under different wave force

$$
(C L=4.5 \mathrm{~m}, \omega=0.4 \mathrm{rad} / \mathrm{s})
$$

From the topological analysis, when the wave force excitation reaches the threshold, the system is in an unstable and chaotic state, and the phenomenon of multi-value and bifurcation of rolling will appear. We can track the trail of a point in motion plane to analyze this phenomenon. Point a is selected for example, when the transverse spacing CL of the trimaran vessel is $4.5 \mathrm{~m}$, and its initial state is $(1.45,-0.2)$, as shown in the Fig.18-(c). The trail of point $\mathrm{a}$ are obtained by solving the nonlinear motion equation (1) with numerical method.

As shown in Fig.20-(a), when the wave force excitation is less than the threshold, the motion is attracted to the equilibrium point $x_{1}$ from the initial point a and makes stable resonant motion. when the wave force excitation exceeds the threshold value, as shown in the Fig.20(b), it can be found that the trail starting from point a escapes from the stable safety region, and a large roll motion and capsizing occurs. Obviously, the trial is attracted by the attractor corresponding to the equilibrium point $x_{4}$ in the phase plane, and the detailed value of $x_{4}$ is listed in Table3. In addition, to continue to increase the wave force excitation, the trail starting from point a has already lost the significance of discussion in the actual ship concept, and the trimaran vessel has already capsized. However, from the perspective of theoretical research, based on the bifurcation topological theory, we can find in Fig.20-(c) that when the excitation exceeds a certain level, the motion begins to be bifurcated and multi-valued. The ship will move back and forth between the two attractive fields of $x_{4}$ and $x_{5}$, and the rolling system will be in a strong chaotic situation.

\section{Chaotic analysis in irregular waves}

In the actual sea environment, the waves are irregular and random, so the deterministic method under regular wave state can no longer be used. Based on the Melnikov function theory of deterministic systems, stochastic dynamics can be studied from the perspective of statistical 
Yihan Zhang, Ping Wang, Yachong Liu, Jingfeng $\mathrm{Hu}$.
Nonlinear rolling stability and chaos research of trimaran vessel with variable lay-outs in regular and irregular waves under wind load

analysis. Melnikov random theory is a statistical extension of the simple zero-point solution of Melnikov function for deterministic systems. Combined with phase flow function and phase space transfer rate, the global stability of stochastic systems can be analyzed.

\subsection{Stochastic Melnikov process and phase space transfer rate}

For Hamilton single degree of freedom system with bounded weak disturbance, using small parameter $\varepsilon$, the motion equation can be expressed as:

$$
\left\{\begin{array}{c}
\dot{X}=\frac{\partial H}{\partial Y} \\
\dot{Y}=\frac{\partial H}{\partial X}-\varepsilon c(X, Y) \frac{\partial H}{\partial Y}+\varepsilon f(X, Y) \xi(t)
\end{array}\right.
$$

The Hamilton Value is:

$$
H=\frac{1}{2} y^{2}+U(x)
$$

Where, $\xi(t)$ is the bounded stochastic excitation. The Hamilton system has hyperbolic saddle points and homoclinic or heteroclinic orbits. $x_{0}(t), y_{0}(t)$.

Based on the deterministic Melnikov function of equation (26), the stochastic Melnikov process can be expressed as:

$$
M\left(t_{0}\right)=\int_{-\infty}^{+\infty} \frac{\partial H}{\partial Y}\left[-c(X, Y) \frac{\partial H}{\partial Y}+f(X, Y) \xi\left(t+t_{0}\right)\right] d t
$$

According to the previous research, for a deterministic system, the chaotic threshold of external excitation can be calculated by solving the first-order zero of Melnikov function, but the first-order zero of stochastic Melnikov process cannot be solved. In order to quantitatively reflect the damage to the stability of the stochastic system, Frey and Jiang further extended the Melnikov method and introduced the phase flow function and phase space transfer rate to describe the instability of the random system [29-30]. Phase flow function $\phi$ is the flow from stable domain into unstable domain in the phase space. The phase space transfer rate refers to the area where the phase flow function of the system flows from the safe domain to the unsafe domain within a unit period of motion or excitation period. When the phase flow function $\phi>$ 0 , system lost stability into chaotic state. Therefore, the external excitation threshold of the stochastic system can be solved by solving the zero of the phase flow function.

The phase flow function is defined as:

$$
\phi=\varepsilon \psi+O\left(\varepsilon^{2}\right)
$$

Where, $\psi$ is average value of Melnikov function. According to equation (45), the Melnikov process of the random system can be expressed as:

$$
M\left(t_{0}\right)=\int_{-\infty}^{+\infty}\left[f(X, Y)\left(\frac{\partial H}{\partial Y}\right) \xi\left(t+t_{0}\right)\right] d t-\int_{-\infty}^{+\infty}\left[c(X, Y)\left(\frac{\partial H}{\partial Y}\right)^{2}\right] d t=M_{z}-M_{d}
$$

Then, the phase space transfer rate of the system is:

$$
\bar{\phi}=\lim _{T \rightarrow \infty} \frac{\varepsilon}{2 T} \int_{-T}^{T}\left[M_{Z}(\xi)-M_{d}\right]^{+} d \xi
$$

Define the function:

$$
f^{+}(\xi)= \begin{cases}f(\xi) & f(\xi)>0 \\ 0 & f(\xi) \leq 0\end{cases}
$$

According to the stationary random characteristics of ergodic states of random waves, the mean time results can be described by statistical means. 


$$
\begin{aligned}
& \bar{\phi}=\lim _{T \rightarrow \infty} \frac{\varepsilon}{2 T} \int_{-T}^{T}\left[M_{z}(\xi)-M_{d}\right]^{+} d \xi=E\left\{\left[M_{z}(\xi)-M_{d}\right]^{+}\right\}=\int_{M_{d}}^{+\infty}\left(v-M_{d}\right) p(v) d v \\
& =\int_{M_{d}}^{+\infty}\left(v-M_{d}\right) \frac{1}{\sqrt{2 \pi} \sigma_{z}} \exp \left(-\frac{v^{2}}{2 \sigma_{z}^{2}}\right) d v
\end{aligned}
$$

Where, $p(v)=\frac{1}{\sqrt{2 \pi} \sigma_{z}} \exp \left(-\frac{v^{2}}{2 \sigma_{z}{ }^{2}}\right)$ is the normal distribution probability density function of the random excitation.

$$
\sigma_{z}=\sqrt{E\left[M_{z}^{2}(\xi)\right]}
$$

Further simplify and set $u=v / \sigma_{z}$, so,

$$
\begin{gathered}
\bar{\phi}=\sigma_{z} \int_{M_{d} / \sigma_{z}}^{+\infty}\left(u-\frac{M_{d}}{\sigma_{z}}\right) \frac{1}{\sqrt{2 \pi}} \exp \left(-\frac{u^{2}}{2}\right) d u \\
=-\sigma_{z} \int_{\frac{M_{d}}{\sigma_{z}}}^{+\infty} u \frac{1}{\sqrt{2 \pi}} \exp \left(-\frac{u^{2}}{2}\right) d u-\int_{\frac{M_{d}}{\sigma_{z}}}^{+\infty}\left(\frac{M_{d}}{\sqrt{2 \pi}}\right) \exp \left(-\frac{u^{2}}{2}\right) d u \\
=-\frac{\sigma_{z}}{\sqrt{2 \pi}} \exp \left[-\frac{1}{2}\left(\frac{M_{d}}{\sigma_{z}}\right)^{2}\right]-M_{d}\left[1-P_{n}\left(\frac{M_{d}}{\sigma_{z}}\right)\right]=\sigma_{z} p_{n}\left(\frac{M_{d}}{\sigma_{z}}\right)+M_{d} P_{n}\left(\frac{M_{d}}{\sigma_{z}}\right)-M_{d}
\end{gathered}
$$

Where, function $p_{n}(x)=\frac{1}{\sqrt{2 \pi}} \exp \left(-\frac{x^{2}}{2}\right)$ is the probability density function of the standard normal distribution, and function $P_{n}(x)=\int_{-\infty}^{x} \frac{1}{\sqrt{2 \pi}} \exp \left(-\frac{x^{2}}{2}\right) d x$ is the probability distribution function of the standard normal distribution.

When $\bar{\phi}=\sigma_{z} p_{n}\left(\frac{M_{d}}{\sigma_{z}}\right)+M_{d} P_{n}\left(\frac{M_{d}}{\sigma_{z}}\right)-M_{d}=0$, it can be considered as the zero of the phase space transfer rate of the stochastic system, and then the threshold of external excitation can be solved.

\subsection{Extreme significant wave height}

The nonlinear roll motion equation of the trimaran vessel in crosswind and random beam waves can be expressed as:

$$
\left\{\begin{array}{c}
\dot{x}=y \\
\dot{y}=f_{\text {wind } 0}\left(1-\frac{x^{2}}{2}+\frac{x^{4}}{24}\right)-\varepsilon\left(\bar{d}_{1} y+\bar{d}_{3} y^{3}-f_{\text {wave } 0} \zeta(t)\right)-\left(r_{1} x+r_{3} x^{3}+r_{5} x^{5}\right)
\end{array}\right.
$$

Where, $\zeta(t)$ is the wave height at time $\mathrm{t}, f_{\text {wave } 0}=F_{\text {wave } 0} /(J+\Delta J), F_{\text {wave } 0}$ is wave force of unit wave amplitude.

According to equation (47), the random Melnikov process of the roll system is:

$$
\begin{gathered}
M\left(t_{0}\right)=\int_{-\infty}^{+\infty} y(t)\left[-\left(\bar{d}_{1} y(t)+\bar{d}_{3} y^{3}(t)\right)+f_{\text {wave } 0} \zeta\left(t+t_{0}\right)\right] d t \\
=-\int_{-\infty}^{+\infty} y(t)\left[\left(\bar{d}_{1} y(t)+\bar{d}_{3} y^{3}(t)\right)\right] d t+\int_{-\infty}^{+\infty} y(t)\left[f_{\text {wave } 0} \zeta\left(t+t_{0}\right)\right] d t=-M_{d}+M_{z}
\end{gathered}
$$

According to the ergodic properties of random waves,

$$
E\left[M_{z}\right]=0
$$

So,

$$
E\left[M_{0}\right]=-E\left[M_{d}\right]<0
$$


Therefore, for the random system of the nonlinear roll of the trimaran, Melnikov simple zero cannot be solved, and the phase space transfer rate is needed to get the Extreme significant wave height.

The mean square value $E\left[M_{z}{ }^{2}\right]$ needs to be solved in the phase space transfer rate. Take the Fourier transform of $M_{z}$, and get the spectrum of $M_{z}$ :

$$
\begin{gathered}
M_{z}(\Omega)=\frac{1}{2 \pi} \int_{-\infty}^{+\infty}\left(\int_{-\infty}^{+\infty} y(t)\left[f_{\text {wave } 0} \zeta\left(t+t_{0}\right)\right] d t\right) e^{-j \Omega t_{0}} d t_{0} \\
=\frac{1}{2 \pi} \int_{-\infty}^{+\infty} y(t)\left(\int_{-\infty}^{+\infty} f_{\text {wave } 0} \zeta(\tau) e^{-j \Omega \tau} d \tau\right) e^{-j \Omega t} d t \\
=2 \pi f_{\text {wave } 0} \zeta(\Omega) y(\Omega)
\end{gathered}
$$

Where, $\zeta(\Omega)$ is the spectral density function of random wave surface elevation, $y(\Omega)$ is the spectral density function of homoclinic(heteroclinic) orbit. The power spectrum function of $M_{z}$ is:

$$
\begin{aligned}
S_{M_{Z}}(\Omega)=2 \pi M_{z}(\Omega) \cdot \operatorname{cong}\left[M_{z}(\Omega)\right]= & 2 \pi[(2 \pi \zeta(\Omega) y(\Omega))] \cdot \operatorname{cong}[(2 \pi \zeta(\Omega) y(\Omega))] \\
& =2 \pi f_{\text {wave } 0} S_{y}(\Omega) S_{\zeta}(\Omega)
\end{aligned}
$$

So, the mean square value of $M_{z}$ can be expressed as:

$$
E\left[M_{z}^{2}\right]=2 \pi f_{\text {wave } 0}^{2} \int_{-\infty}^{+\infty} S_{y}(\Omega) S_{\zeta}(\Omega) d \Omega={\sigma_{z}}^{2}
$$

ITTC double - parameter spectrum is used as the wave spectrum.

$$
S_{\zeta}(\Omega)=\frac{124 H_{S}^{2}}{T_{z} \Omega^{5}} \exp \left(\frac{-496}{T_{z}^{4} \Omega^{4}}\right)
$$

Where, $H_{s}$ is the significant wave height, and $T_{z}$ is the mean span zero period. It is easy to find that $\sigma_{z}$ is proportional to $H_{s}$. So, $\sigma_{z}=\sigma_{z 0} H_{s}$, and $\sigma_{z 0}$ is the mean square value under unit significant wave height.

Then, The phase space transfer rate in equation (52) is converted to:

$$
\bar{\phi}=\sigma_{z 0} H_{s} p_{n}\left(\frac{M_{d}}{\sigma_{z 0} H_{s}}\right)+M_{d} P_{n}\left(\frac{M_{d}}{\sigma_{z 0} H_{s}}\right)-M_{d}
$$

When $H_{s} \rightarrow \infty, \quad \bar{\phi}=\frac{1}{\sqrt{2 \pi}} \sigma_{z 0} H_{s}-\frac{1}{2} M_{d}$

If $\bar{\phi}=0$, the extreme significant wave height $H_{s c r}$ can be obtained.

$$
H_{s c r}=\frac{\sqrt{2 \pi} M_{d}}{2 \sigma_{z 0}}=\frac{\varepsilon \sqrt{2 \pi} M_{d}}{\varepsilon 2 \sigma_{z 0}}
$$

When the significant wave height exceeds $H_{s c r}$, the rolling system will lose its stability in irregular waves and enter chaos state. For different layouts, the trimaran vessel with transverse spacing $\mathrm{CL}=4.5 \mathrm{~m}$ was selected as the research object.

For the numerator part in equation (61):

$$
\varepsilon M_{d}=\int_{-\infty}^{+\infty} y(t)\left[\left(d_{1} y(t)+d_{3} y^{3}(t)\right)\right] d t
$$

This integral is similar to the solution in deterministic system. Since the homoclinic (heteroclinic) orbit $y(t)$ cannot be solved analytically, numerical integral solution is applied. For the denominator part in equation (62):

$$
\varepsilon \sigma_{z 0}=\sqrt{2 \pi} f_{\text {wave } 0} \sqrt{\int_{-\infty}^{+\infty} S_{y}(\Omega) S_{\zeta_{0}}(\Omega) d \Omega}
$$


Where, $S_{\zeta_{0}}(\Omega)$ is the wave energy spectrum of unit significant wave height. $f_{\text {wave } 0}$ is the wave force of unit wave amplitude, which is calculated by 3D potential theory. Fig.21 shows the wave force in frequency domain by 3D potential theory.

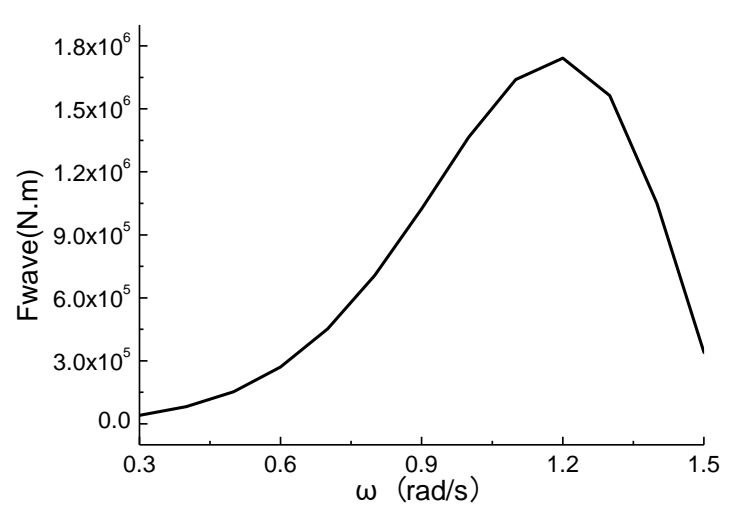

Fig.21 Wave force response curve in frequency domain

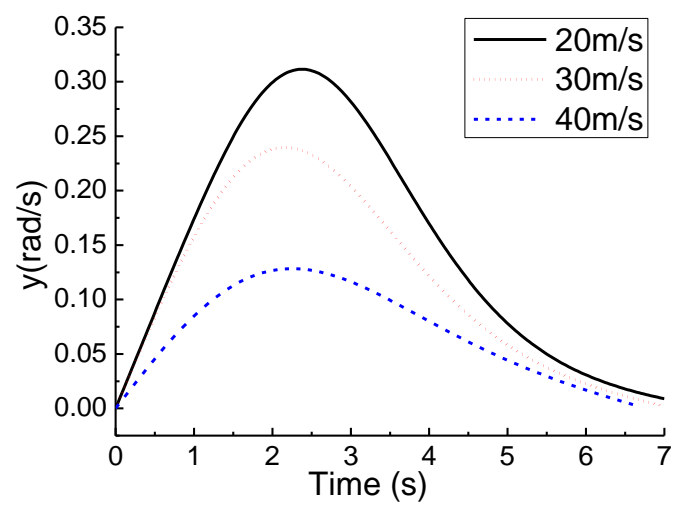

Fig.22 $y(t)$ of different wind speeds $(\mathrm{CL}=4.5 \mathrm{~m})$

$S_{y}(\Omega)$ is the power spectral function of the roll angular velocity $y(t)$ along a homoclinic or heteroclinic orbit. For the high-order nonlinear rolling system of the trimaran vessel, $y(t)$ cannot be solved analytically, so the discrete solution can only be obtained by numerical calculation. The Fig. 22 shows the discrete orbit equation of $y(t)$ corresponding to different wind speeds obtained by the 4-order Runge Kutta method.

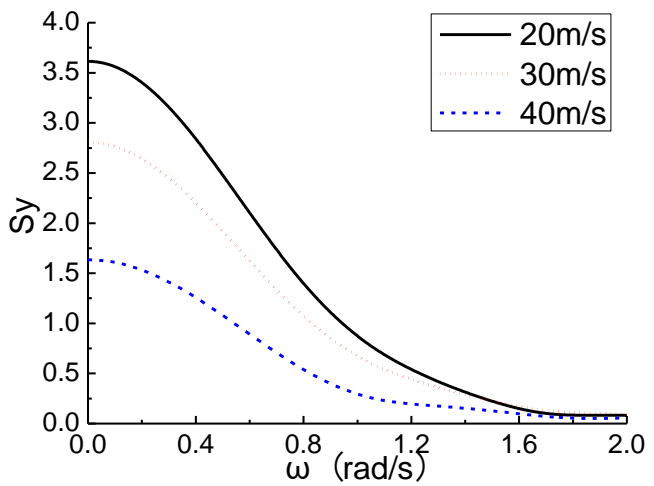

Fig.23 $S y(\Omega)$ of different wind velocity $(\mathrm{CL}=4.5 \mathrm{~m})$

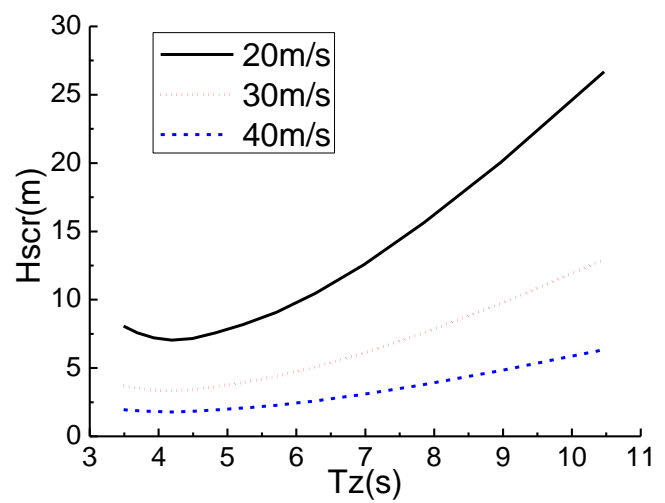

Fig.24 The Hscr of different wind velocity $(\mathrm{CL}=4.5 \mathrm{~m})$

The power spectrum of $y(t)$ can be obtained by Fourier transform. As shown in Fig.23, the power spectrum of different wind speeds in the low-frequency region is different obviously, and the higher the wind speed is, the smaller the power spectrum is. Then, according to equations (63) and (64), the $H_{s c r}$ corresponding to different wave frequencies can be obtained. As shown in Fig.24, with the increase of wind speed, the $H_{s c r}$ when entering the rolling chaos state in the irregular wave gradually decreases. Moreover, with the change of wave period, the $H_{s c r}$ tends to decrease first and then increase. 
Yihan Zhang, Ping Wang, Yachong Liu, Jingfeng $\mathrm{Hu}$.
Nonlinear rolling stability and chaos research of trimaran vessel with variable lay-outs in regular and irregular waves under wind load

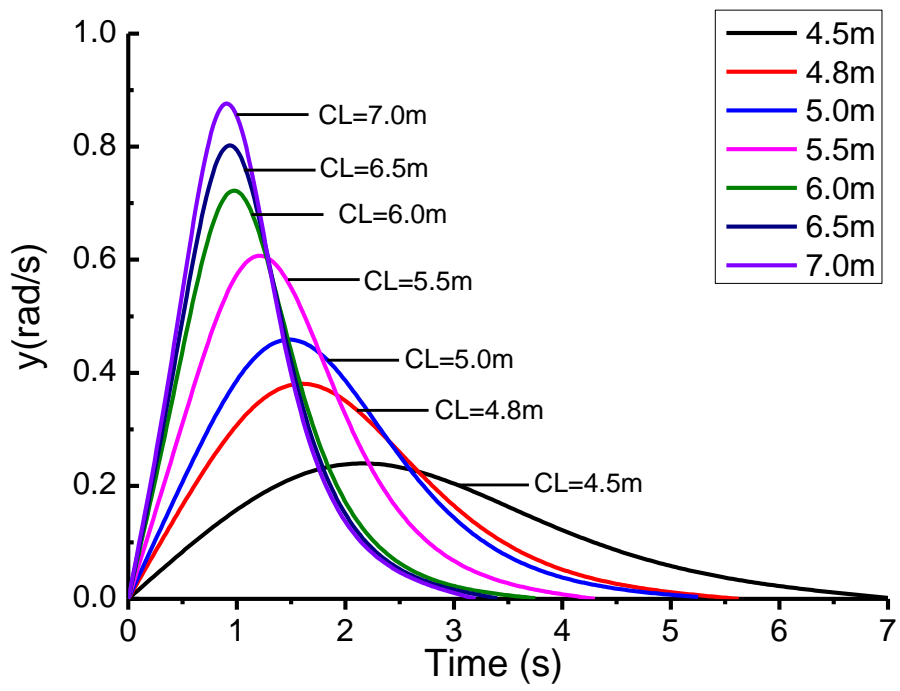

Fig.25 $y(t)$ of different CL (Vwind $=30 \mathrm{~m} / \mathrm{s})$

For the trimaran vessel with different transverse spacing CL, when the wind speed is $30 \mathrm{~m} / \mathrm{s}$, the $y(t)$ is obtained according to the above method. As shown in the Fig.25, the trimaran vessel with large transverse spacing has lager area bounded by the homoclinic orbit. Fig.26 shows power spectrum of $y(t)$ corresponding to different transverse spacing, and the Fig.27 shows $H_{s c r}$ of different transverse spacing. It can be clearly found that the trimaran vessel with large transverse spacing has a lager $H_{s c r}$, the stronger the stability of the rolling motion in the irregular wave is, which is consistent with the conclusion in the regular wave. It also means that the roll motion in irregular waves is more stable, which is consistent with the conclusion in regular waves. Combined with the actual sea conditions, the trimaran ship studied in this paper is prone to unstable rolling in the region with a small wave period. In the condition of $30 \mathrm{~m} / \mathrm{s}$ of the cross wind, the minimum $H_{s c r}$ of a trimaran vessel with $\mathrm{CL}=7.0 \mathrm{~m}$ is about $12 \mathrm{~m}$, so it will not encounter such sea conditions in its sailing sea area, but unstable rolling may occur in severe weather such as typhoon, and it's even more dangerous for trimarans of small CL. From the point of view of limitation, a single-hull ship can be considered as a trimaran with a very small transverse spacing. Therefore, the rolling stability of trimaran vessels with the same tonnage and the same center of gravity is obviously better than that of mono-hull ships in theory.

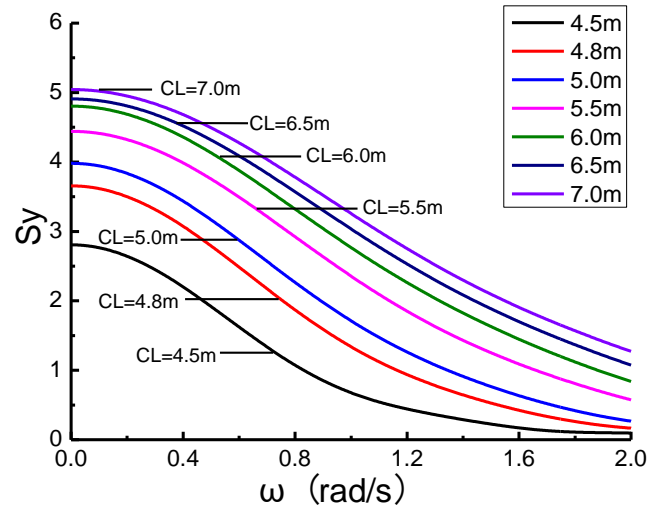

Fig.26 $S y(\Omega)$ of different CL(Vwind=30m $/ \mathrm{s})$

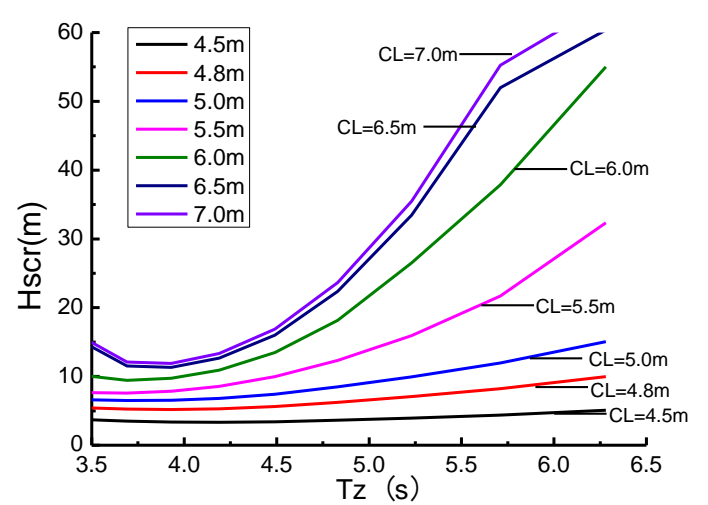

Fig.27 The Hscr of different CL (Vwind=30m/s)

\section{Conclusions}

It is significant to study the stability and chaos of roll motion for the trimaran vessel with the variation of layout, which is the main parameter in the design of the trimaran vessel. In 
rolling system, both damping coefficients and restoring moment coefficients are important for the stability analysis. With the CFD method and high-order polynomial fitting of GZ curves, the characteristics of the damping coefficients and restoring moment coefficients are found, which has the deep relationship with the chaotic analysis.

In the phase portrait analysis, with the increase of the transverse spacing, the stable attraction domain gradually expands, and the saddle point disappears after reaching a certain distance, and the system reaches the state of global stability. After the wind load is added, the system has the asymmetric migration, and the heteroclinic orbits surrounding the stability region change topologically, from heteroclinic orbits to homoclinic orbits, and the equilibrium point also shifts accordingly with the decrease of the stability. Based on the Lyapunov stability theory and Melnikov method, the wave force threshold of rolling chaos for trimaran vessel is calculated in regular waves. The wave force threshold increases gradually with the increase of transverse spacing, indicating the enhancement of system stability. We also find that the nonlinear roll damping is sensitive to wave force threshold. The increase of the restoring force will increase the area of the safety region and increase the external excitation of chaos. The Lyapunov exponent and safe basin analysis verifies the threshold by Melnikov method. The extreme significant wave height of the trimaran vessel is calculated by using the phase space transfer rate in irregular waves, which can be the reference to the design of the trimaran vessel.

\section{ACKNOWLEDGEMENT}

We would like to thank the anonymous referee for their valuable comments and suggestions. The authors gratefully thank the professors and staff in Marine Design \& Research Institute of China for the given support. The authors also thank the Harbin Engineering University for the valuable support during the evaluation of the model test results. This study is supported by the Ship Advance Research Project. (30203020101) and the National Natural Science Foundation of China (51709030).

\section{REFERENCES}

[1] Bačkalov I, Bulian G, Cichowicz J, et al.Ship stability, dynamics and safety: Status and perspectives from a review of recent STAB conferences and ISSW events. Ocean engineering. 2016; 116: 312-349. https://doi.org/10.1016/j.oceaneng.2016.02.016

[2] Hayashi C. Nonlinear oscillations in physical systems, McGraw-Hill, New York; reprinted University Press, Princeton, 1985.

[3] Maki A, Virgin LN, et al. On the lossof stability of periodic oscillations and its relevance to ship capsize, Journal of Marine Scienceand Technology, 2019; 24(3):846-854. https://doi.org/10.1007/s00773-0180591-X

[4] Holmes PJ. and Rand DA. The bifurcation of Duffing's equation: An application of catastrophe theory, Journal of Sound and Vibration, 1976; 44(2):237-253. https://doi.org/10.1016/0022-460X(76)90771-9

[5] Parker TS, Chua L. Practical numerical algorithms for chaotic systems. Springer Science\&Business Media.2012.

[6] Shi Y. Melnikov analysis of chaos in a simple SIR model with periodically or stochastically modulated nonlinear incidence rate. Journal of Biological Dynamics. 2020; 14(1):269-288. https://doi.org/10.1080/17513758.2020.1718222

[7] Virgin LN. The nonlinear rolling response of a vessel including chaotic motions leading to capsize in regular seas. Applied Ocean Research. 1987; 9(2): 331-352. https://doi.org/10.1016/0141-1187(87)90011$\underline{3}$

[8] Virgin LN. On the harmonic response of an oscillator with unsymmetric restoring force, Journal of Sound and Vibration, 1988; 126(1): 157-165. https://doi.org/10.1016/0022-460X(88)90405-1

[9] Virgin LN. Approximate criterion for capsize based on deterministic dynamics, Dynamics and Stability of Systems, 1989; 4(1):55-70. https://doi.org/10.1080/02681118908806062 
Yihan Zhang, Ping Wang, Yachong Liu, Jingfeng $\mathrm{Hu}$.
Nonlinear rolling stability and chaos research of trimaran vessel with variable lay-outs in regular and irregular waves under wind load

[10] Holmes PJ. Averaging and chaotic motions in forced oscillations, SIAM Journal on AppliedMathematics, 1980; 38(1):65-80. https://doi.org/10.1137/0138005

[11] Greenspan B and Holmes P, Repeated resonance and homoclinic bifurcation in a periodically forced family of oscillators, SIAM journal on mathematical analysis, 1984; 15(1):69-97. https://doi.org/10.1137/0515003

[12] Thompson JMT, Bishop S, Leung L. Fractal basins and chaotic bifurcations prior to escape from a potential well. Physics Letters A. 1987;121(3):116-120. https://doi.org/10.1016/0375-9601(87)90403-8

[13] Falzarano JM. Predicting complicated dynamics leading to vessel capsizing: [Phd dissertation], Michigan: the University of Michigan.1990.

[14] Nayfeh AH, Balachandran B. Applied nonlinear dynamics: Analytical, Computational, and Experimental Methods. Wiley Series in Nonlinear Science. 1995;356-537. https://doi.org/10.1002/9783527617548

[15] Maki A, Umeda $\mathrm{N}$ and Ueta $\mathrm{T}$. Melnikov integral formula for beam sea roll motion utilizing a nonHamiltonian exact heteroclinic orbit. Journal of marine science and technology, 2010;15(1):102-106. https://doi.org/10.1007/s00773-009-0076-Z

[16] Wan W, Leigh McCue. Application of the extended Melnikov's method for single-degree-of-freedom vessel roll motion. Ocean Engineering. 2008; https://doi.org/10.1016/j.oceaneng.2008.08.011

[17] Surendran S, Lee SK, Reddy JVR, Lee G. Non-linear roll dynamics of a ro-ro ship in waves. Ocean Engineering. 2005; 32(14-15), 1818-1828. https://doi.org/10.1016/j.oceaneng.2004.11.014

[18] Dostal L, Kreuzer E and Namachchivaya N Sri, Non-standard stochastic averaging of large-amplitude ship rolling in random seas, Proceedings of the Royal Society A: Mathematical, Physical and Engineering Sciences, 2012; 468(2148): 4146-4173. https://doi.org/10.1098/rspa.2012.0258

[19] Nekrasov VA. Probabilistic problem of seakeeping. Sudostroenie, Leningard(in Russian)On-board intelligence system.1978.

[20] Haddara MR. A modified approach to the application of the Fokker-Planck equation to nonlinear ship motions in random waves. International shipbuilding progress.1974;21(242):283-288. https://doi.org/10.3233/ISP-1974-2124201

[21] Roberts JB.A stochastic theory for nonlinear ship rolling in irregular seas. Journal of ship research. 1982;26(04):229-245. https://doi.org/10.5957/jsr.1982.26.4.229

[22] Roberts JB, Vasta M. Markov modelling and stochastic identification for nonlinear ship rolling in random waves. Philosophical Transaction of the Royal Society of London. Series A: Mathematical, Physical and Engineering Sciences. 2000; 358(1771):1917-1941. https://doi.org/10.1098/rsta.2000.0621

[23] Roberts JB, Dunne JF, Debonos A. Stochastic estimation methods for non-linear ship roll motion. Probabilistic Engineering Mechanics.1994;9(1-2):83-93. https://doi.org/10.1016/0266-8920(94)90032-9

[24] Chai W, Naess Arvid, Leira BJ. Influence of linear filters and nonlinear damping models on the stochastic roll response of a ship in random seas. Proceedings of the 12th International Conference on the Stability of Ships and Ocean Vehicles. 2015;1-10.

[25] Chai W, Naess, et al. Long-Term Extreme Response and Reliability of a Vessel Rolling in Random Beam Seas. Journal of Offshore Mechanics \& Arctic Engineering. 2018. https://doi.org/10.1115/1.4037789

[26] Maki A. Estimation method of the capsizing probability in irregular beam seas using non-gaussian probability density function, Journal of Marine Science and Technology,2017; 22(2): 351-360. https://doi.org/10.1007/s00773-016-0415-9

[27] Ren ZC, Xu W, Wang DL. Dynamic and first passage analysis of ship roll motion with inelastic impacts via path integration method. Nonlinear dynamics.2019;97:391-402. https://doi.org/10.1007/s11071-01904975-X

[28] Li SH, Wang KK. Chaos analysis of ship rolling motion in stochastic beam seas. Journal of Vibroengineering. 2017; 19(8):6403-6411. https://doi.org/10.21595/jve.2017.17507

[29] Frey M, Simiu E. Noise-induced chaos and phase space flux. Physica D: Nonlinear Phenomena.1993; 63(34):321-340. https://doi.org/10.1016/0167-2789(93)90114-G

[30] Jiang CB, Troesch AW, Shaw W. Highly nonlinear rolling motion of biased ships in random beam seas. Journal of Ship Research.1996; 40(2): 125-135. https://doi.org/10.5957/jsr.1996.40.2.125

[31] Liu LQ. Study on the nonlinear random dynamic behaviors of ship roll [Phd dissertation]. Tianjin University, 2006, China.

[32] Zhang J W, Wu W Q, Hu J Q. Study on the safety degree of ship capsizing in stochastic waves[J]. Journal of Ship Production and Design, 2017, 33(1):24-30. https://doi.org/10.5957/jspd.2017.33.1.24 
[33] Onas AS, Datla R. Experimental analysis of roll damping of a trimaran with varying side hull stagger and transverse spacing. Proceedings from World Maritime Technology Congress, Mumbai, India. 2019:212218.

[34] Wang SM, Ma S, Duan WY. Seakeeping optimization of trimaran outrigger layout based on NSGA-II. Applied Ocean Research. 2018; 78:110-122. https://doi.org/10.1016/j.apor.2018.06.010

[35] Ghadini P, Nazemian A. Numerical scrutiny of the influence of side hulls arrangement on the motion of a Trimaran vessel in regular waves through CFD analysis. Journal of the Brazilian Society of Mechanical Science and Engineering. 2019; 41(1):1-10. https://doi.org/10.1007/s40430-018-1505-X

[36] Zhang JW. Investigation into roll damping of trimaran ships. NARG Report. 1996; 96(1026):225-276

[37] Onas AS, Datla R. Non-linear roll motions of a frigate-type trimaran. 11th International Conference on Fast Sea Transportation FAST, Honolulu, Hawaii, USA. 2011; 607-614.

[38] Zhang JW, Andrews DJ. Roll damping characteristics of a trimaran displacement ship. International Shipbuilding Progress. 1999; 46(448): 445-472.

[39] Yildiz B, Sener B, Yurtseven A, Katayama T. Numerical and experimental calculation of roll amplitude effect on roll damping. Brodogradnja. 2019, 70(2): 1-15. https://doi.org/10.21278/brod70201

[40] Song SS, Kim SH, Paik KJ. Determination of linear and nonlinear roll damping coefficients of a ship section using CFD. Brodogradnja. 2019, 70(4): 17-33. https://doi.org/10.21278/brod70402

[41] Wassermann S, Feder DF, Abdel-Maksoud M. Estimation of ship roll damping-A comparison of the decay and the harmonic excited roll motion technique for a post panamax container ship. Ocean Engineering. 2016;120: 371-382. https://doi.org/10.1016/j.oceaneng.2016.02.009

[42] Bikdash M, Balachandran B, Navfeh A. Melnikov analysis for a ship with a general roll-damping model. Nonlinear Dynamics.1994; 6(1): 101-124

[43] Zhang YH, Wang P, Lai YP, and Liu YP. Prediction of nonlinear roll damping for high speed trimaran ship in viscous flow. Shipbuilding of China. 2017; 58(3): 23-32.

[44] Senjanovic I, Cipric G, Parunov J. Survival analysis of fishing vessels rolling in rough seas. Philosophical Transactions: Mathematical, Physical and Engineering Sciences. 2000;358 (1771): 1943-1965. https://doi.org/10.1098/rsta.2000.0622

[45] Li JD. Ship Seakeeping Performance. Harbin Engineering University, China. 1991.

[46] Udwadia FE, Hubertus F. An efficient and stable approach for computation of Lyapunov characteristic exponents of continuous dynamical systems. Applied Mathematics and Computation. 2001;121(2): 219259. https://doi.org/10.1016/S0096-3003(99)00292-1

[47] Thompson JMT. Chaotic phenomena triggering the escape from a potential well. Proceedings of the Royal Society of London A: Mathematical, Physical and Engineering Sciences. 1989; 421(1861): 195-225. https://doi.org/10.1098/rspa.1989.0009

[48] Thompson JMT, Rainey RCT, Soliman MS. Ship stability criteria based on chaotic transients from incursive fractals. Philosophical Transactions of the Royal Society of London A: Mathematical, Physical and Engineering Sciences.1990; 332(1624): 149-167. https://doi.org/10.1098/rsta.1990.0106

[49] Spyrou K, Cotton B and Gurd B. Analytical expressions of capsize boundary for a ship with roll bias in beam waves, Journal of ship research, 2002; 46(3): 167-174. https://doi.org/10.5957/jsr.2002.46.3.167

\begin{tabular}{|c|c|c|}
\hline Submitted: & 24.07.2021. & Yihan Zhang, zhangyihan437@163.com \\
\hline & & Marine Design \& Research Institute of China, China, 200011. \\
\hline & & Ping Wang, hongliu@shmtu.edu.cn \\
\hline Accepted: & 29.09.2021. & Marine Design \& Research Institute of China, China, 200011. \\
\hline & & Yachong Liu, yachongliu@163.com \\
\hline & & Marine Design \& Research Institute of China, China, 200011. \\
\hline & & Jingfeng Hu, huakejf1992@126.com \\
\hline & & Marine Design \& Research Institute of China, China, 2000 \\
\hline
\end{tabular}

